\title{
An Impulsive Three-Species Model with Square Root Functional Response and Mutual Interference of Predator
}

\author{
Zhen Wang, Yuanfu Shao, Xianjia Fang, and Xiangmin Ma \\ School of Science, Guilin University of Technology, Guilin, Guangxi 541004, China \\ Correspondence should be addressed to Yuanfu Shao; shaoyuanfu@163.com
}

Received 26 January 2016; Accepted 27 April 2016

Academic Editor: Ryusuke Kon

Copyright (C) 2016 Zhen Wang et al. This is an open access article distributed under the Creative Commons Attribution License, which permits unrestricted use, distribution, and reproduction in any medium, provided the original work is properly cited.

\begin{abstract}
An impulsive two-prey and one-predator model with square root functional responses, mutual interference, and integrated pest management is constructed. By using techniques of impulsive perturbations, comparison theorem, and Floquet theory, the existence and global asymptotic stability of prey-eradication periodic solution are investigated. We use some methods and sufficient conditions to prove the permanence of the system which involve multiple Lyapunov functions and differential comparison theorem. Numerical simulations are given to portray the complex behaviors of this system. Finally, we analyze the biological meanings of these results and give some suggestions for feasible control strategies.
\end{abstract}

\section{Introduction and Model Formulation}

In real world, the study on models of three or more species is very popular, such as food-chain and food webs systems, which have extremely rich dynamics $[1,2]$. For predator-prey model, in portrayal of the relationship between predator and prey, a crucial element is the classic definition of a predator's functional response. In the past few decades, many different functional responses have been extensively investigated [37]. For example, Liu et al. [5] gave the following Holling type II functional response which describes the relations of one prey and one predator:

$$
\begin{aligned}
& x_{1}^{\prime}(t)=x_{1}(t)\left(a-b x_{1}(t)\right)-\frac{\alpha x_{1}(t) x_{2}(t)}{1+w x_{1}(t)}, \\
& x_{2}^{\prime}(t)=x_{2}(t)\left(\frac{k \alpha x_{1}(t)}{1+w x_{1}(t)}-c\right),
\end{aligned}
$$

where $x_{1}(t)$ is the density of prey and $x_{2}(t)$ is the density of predator at time $t$. $a$ is the intrinsic growth rate of prey. $b$ represents the rate of intraspecific competition or density dependence. $c$ is the death rate of predator. $k$ is transformation rate for the predator to prey; $\alpha x_{1}(t) x_{2}(t) /(1+$ $\left.w x_{1}(t)\right)$ denotes the Holling type II functional response.

However, in the actual ecosystem, if examining more complicated ecological case, some preys show herd behavior.
That is to say, the predator interacts with the prey along the outer corridor of the herd of prey. Hence actual dynamic behaviors of individuals have not been described in detail by the predation term of Holling type II functional response. Ajraldi et al. [8] pointed out that, by using the terms of the square root of the prey population, the response functions of prey that exhibited herd behavior are more properly modeled. In this respect, Braza [9] gave the following predator-prey model:

$$
\begin{aligned}
& x_{1}^{\prime}(t)=x_{1}(t)\left(a-b x_{1}(t)\right)-\frac{\alpha \sqrt{x_{1}(t)} x_{2}(t)}{1+w \sqrt{x_{1}(t)}}, \\
& x_{2}^{\prime}(t)=x_{2}(t)\left(\frac{k \alpha \sqrt{x_{1}(t)}}{1+w \sqrt{x_{1}(t)}}-c\right),
\end{aligned}
$$

where $\alpha \sqrt{x_{1}(t)} /\left(1+w \sqrt{x_{1}(t)}\right)$ is the square root functional response. $\mathrm{Ma}$ et al. [10] also investigated a predator-prey system with square root functional response. Their results showed that square root functional response brought about large influence to the dynamical behaviors.

On the other hand, few researchers consider the mutual interference between predators, but mutual interference between predators always exists in the actual ecosystem. In 1971, Hassell set about studying the capturing behavior between hosts and parasites; he discovered that hosts or 
parasites had the tendency to depart from each other when they met, which affected the hosts capturing. If the size of parasite became larger and larger, then the mutual interference would be stronger and stronger. Hence he introduced the mutual interference of predator [11]. Considering the effect from mutual interference between predators, the dynamic behaviors were more complex. For example, He et al. [12] and Zhang et al. [13] investigated the mutual interference of the predator in detail and obtained much different dynamics with those models without mutual interference. Hence, for predator-prey system, it is necessary to consider the mutual interference of predator.

Based on above discussion, we give the following preypredator system with square root functional response and mutual interference of the predator:

$$
\begin{aligned}
& x_{1}^{\prime}(t)=x_{1}(t)\left(a-b x_{1}(t)\right)-\frac{\alpha \sqrt{x_{1}(t)} x_{2}(t)^{m}}{1+w \sqrt{x_{1}(t)}}, \\
& x_{2}^{\prime}(t)=\frac{k \alpha \sqrt{x_{1}(t)} x_{2}(t)^{m}}{1+w \sqrt{x_{1}(t)}}-c x_{2}(t),
\end{aligned}
$$

where $0<m \leq 1$ (see [8] for the details).

As is known to all, insects have a profound impact on the survival and development of human beings. Most insects are beneficial to human beings; only a few insects are harmful to human life and agricultural development when they reach a certain amount. Hence it is necessary to kill the harmful pests or control them in a certain quantity. Chemical control and biological control are two most commonly used methods. Chemical control is often applied by spraying pesticides, which are used widely because they can kill pests quickly and reduce economic losses in a short time, but they also produce serious environmental pollution. For less pollution to the environment, by stocking or releasing natural enemies, biological control appears, but the effects are not very great. In order to combine different approaches to control pests at the same time, integrated pest management is given to maximize control efficiency and reduce pollution. During the last two decades, ecological pest control is a complex project $[14,15]$. For predator-prey system, pest control strategy has been an important topic for many researchers $[16,17]$.

The main purpose of this paper is to investigate the dynamical behaviors of an impulsive one-predator twoprey model with mutual interference, square root functional response, and integrated control methods. The model is described by the following differential equations:

$$
\begin{aligned}
& x_{1}^{\prime}(t) \\
& =x_{1}(t)\left(a_{1}-b_{1} x_{1}(t)-\alpha x_{2}(t)\right) \\
& \quad-\frac{c_{1} x_{3}(t)^{m} \sqrt{x_{1}(t)}}{1+d_{1} \sqrt{x_{1}(t)}}, \\
& x_{2}^{\prime}(t) \\
& =x_{2}(t)\left(a_{2}-b_{2} x_{2}(t)-\beta x_{1}(t)\right) \\
& \quad-\frac{c_{2} x_{3}(t)^{m} \sqrt{x_{2}(t)}}{1+d_{2} \sqrt{x_{2}(t)}},
\end{aligned}
$$

$$
\begin{aligned}
& x_{3}^{\prime}(t) \\
& =-a_{3} x_{3}(t) \\
& +\left(\frac{c_{1} k_{1} \sqrt{x_{1}(t)}}{1+d_{1} \sqrt{x_{1}(t)}}+\frac{c_{2} k_{2} \sqrt{x_{2}(t)}}{1+d_{2} \sqrt{x_{2}(t)}}\right) x_{3}(t)^{m}, \\
& t \neq n T, t \neq(n+l-1) T, \\
& x_{1}\left(t^{+}\right)=\left(1-\mu_{1}\right) x_{1}(t), \\
& x_{2}\left(t^{+}\right)=\left(1-\mu_{2}\right) x_{2}(t), \\
& x_{3}\left(t^{+}\right)=\left(1-\mu_{3}\right) x_{3}(t), \\
& t=(n+l-1) T, \\
& x_{1}\left(t^{+}\right)=x_{1}(t), \\
& x_{2}\left(t^{+}\right)=x_{2}(t), \\
& x_{3}\left(t^{+}\right)=x_{3}(t)+p, \\
& t=n T \text {, }
\end{aligned}
$$

where $x_{1}(t), x_{2}(t)$, and $x_{3}(t)$ are densities of two preys and one predator at time $t$, respectively. $a_{i}(i=1,2)$ is intrinsic increasing rate; $a_{3}$ is the death rate of predator. $m$ represents the mutual interference of the predator: $0<m \leq 1 . k_{i}(i=$ $1,2)$ is transformation rate for the predator to prey. $b_{i}(i=$ $1,2)$ is death rate of prey; $0<\mu_{i}<1(i=1,2,3)$ represents the percent of prey-predator that dies at time $t=(n+l-$ 1)T, $0<l<1 . p>0$ is the releasing number of predators at $t=n T$. Parameters $\alpha, \beta$ are competitive effects between two preys, respectively. Parameter $T$ is the moment period of impulsive effect. The integer $n \in N$; $N$ is the set of all nonnegative integers. All parameters are positive constants.

We aim to investigate the dynamical behaviors of (4). From the biological point of view, we only consider system (4) in the biological meaningful region:

$$
\begin{aligned}
D & =\left\{x_{1}(t), x_{2}(t), x_{3}(t): x_{1}(t) \geq 0, x_{2}(t)\right. \\
& \left.\geq 0, x_{3}(t) \geq 0\right\},
\end{aligned}
$$

and the initial conditions for system (4) are

$$
\begin{array}{ll}
\left(\varphi_{1}(t), \varphi_{2}(t), \varphi_{3}(t)\right) \in C\left([-T, 0], R_{+}^{3}\right), & \varphi_{i}(t)>0 \\
R_{+}^{3}=\left\{\left(x_{1}, x_{2}, x_{3}\right) \mid x_{i} \in R_{+}=[0,+\infty)\right\}, & i=1,2,3 .
\end{array}
$$

The structure of this paper is as follows. In Section 2, we give some definitions, notations, and lemmas. In Section 3, by using techniques of impulsive perturbations, Floquet theory, and comparison theorem, we discuss stability, extinction, and permanence of system (4). We give corollaries for single chemical control in Section 4. Then we give some examples and numerical analysis of system (4) in Section 5. Finally, we conclude this paper with a brief discussion in Section 6 . 


\section{Preliminaries}

In this section, some helpful remarks, notations, definitions, and lemmas are introduced which are useful for our main results.

Let $f=\left(f_{1}, f_{2}, f_{3}\right)$ be the map defined by the righthand sides of (4). Solution of (4), denoted by $X(t)=$ $\left(x_{1}(t), x_{2}(t), x_{3}(t)\right): R_{+} \rightarrow R_{+}^{3}$, is continuously differentiable on $((n-1) T,(n+l-1) T)$ and $((n+l-1) T, n T), \quad(n \in N)$. Let $V: R_{+} \times R_{+}^{3} \rightarrow R_{+}$, and then $V$ is said to belong to class $V_{0}$ if

(i) $V$ is continuous on $((n-1) T,(n+l-1) T] \times R_{+}^{3}$ and $((n+l-1) T, n T] \times R_{+}^{3}, n=1,2, \ldots$, and for each $X(t) \in$ $R_{+}^{3}, \lim _{(t, y) \rightarrow\left((n+l-1) T^{+}, x\right)} V(t, y)=V\left((n+l-1) T^{+}, x\right)$ and $\lim _{(t, y) \rightarrow\left(n T^{+}, x\right)} V(t, y)=V\left(n T^{+}, x\right)$ exist;

(ii) $V$ is locally Lipschitzian in $X(t)$.

Definition 1. Let $V \in V_{0}$. For $(t, x) \in((n-1) T,(n+l-1) T] \times R_{+}^{3}$ and $((n+l-1) T, n T] \times R_{+}^{3}$, the upper right derivative of $V$ with respect to the impulsive differential system (4) is defined as

$$
\begin{aligned}
& D^{+} V(t, x) \\
& \quad=\limsup _{h \rightarrow 0_{+}} \frac{1}{h}[V(t+h, x+h f(t, x))-V(t, x)] .
\end{aligned}
$$

Definition 2. If there exist positive constants $m_{0}$ and $M_{0}$, with each positive solution $\left(x_{1}(t), x_{2}(t), x_{3}(t)\right)$ of (4) satisfying $m_{0} \leq x_{1}(t), x_{2}(t), x_{3}(t) \leq M_{0}$ for all $t$ sufficiently large, then system (4) is said to be permanent.

Remark 3. The solution $X(t)$ of (4) is a piecewise continuous function; $X(t): R_{+} \rightarrow R_{+}^{3}, X(t)$ is continuous on $((n-$ $1) T,(n+l-1) T)$ and $((n+l-1) T, n T)$, and $X\left(t_{0}^{+}\right)=\lim _{t \rightarrow t_{0}^{+}} X(t)$ exists, where $t_{0}=(n+l-1) T^{+}$and $n T^{+}$. The smoothness properties of $f$ ensure the global existence and peculiarity of solutions of (4) (see [18] for the details).

$$
x_{3}^{*}= \begin{cases}\frac{p \exp \left(-a_{3}(t-(n-1) T)\right)}{1-\left(1-\mu_{3}\right) \exp \left(-a_{3} T\right)}, & (n-1) T<t \leq(n+l-1) T, \\ \frac{p\left(1-\mu_{3}\right) \exp \left(-a_{3}(t-(n-1) T)\right)}{1-\left(1-\mu_{3}\right) \exp \left(-a_{3} T\right)}, & (n+l-1) T<t \leq n T,\end{cases}
$$

Lemma 4 (see [18]). Let $V \in V_{0}$, and assume that

$$
\begin{gathered}
D^{+} V(t, X) \leq g(t, V(t, X)), \\
\qquad \neq(n+l-1) T, t \neq n T, \\
V\left(t, X\left(t^{+}\right)\right) \leq \psi_{n}^{1}(V(t, X)), \quad t=(n+l-1) T, \\
V\left(t, X\left(t^{+}\right)\right) \leq \psi_{n}^{2}(V(t, X)), \quad t=n T,
\end{gathered}
$$

where $g: R_{+} \times R_{+} \rightarrow R_{+}$and $g$ is continuous on $((n-1) T,(n+$ $l-1) T] \times R_{+}$and $((n+l-1) T, n T] \times R_{+}$for $u \in R_{+}^{2}, n \in$ $N, \lim _{(t, y) \rightarrow\left(n T^{+}, u\right)} g(t, y)=g\left(n T^{+}, u\right)$ exists, $\psi_{n}^{1}, \psi_{n}^{2}: R_{+} \rightarrow$ $R_{+}$are nondecreasing for all $n \in N$. Let $r(t)$ be the maximal solution of the scalar impulsive differential equation,

$$
\begin{aligned}
& u^{\prime}(t)=g(t, u(t)), \quad t \neq(n+l-1) T, \quad t \neq n T, \\
& u\left(t^{+}\right)=\psi_{n}^{1}(u(t)), \quad t=(n+l-1) T, \\
& u\left(t^{+}\right)=\psi_{n}^{2}(u(t)), \quad t=n T, \\
& u\left(0^{+}\right)=u_{0} \geq 0,
\end{aligned}
$$

existing on $[0, \infty)$. Then $V\left(0^{+}, X_{0}\right) \leq u_{0}$ implies that $V(t, X(t)) \leq r(t)$ for all $t \geq 0$, where $X(t)$ is any solution of system (4).

Next, we introduce some fundamental properties about the following subsystem of (4):

$$
\begin{aligned}
x_{3}^{\prime}(t) & =-a_{3} x_{3}(t), \quad t \neq n T, \quad t \neq(n+l-1) T, \\
x_{3}\left(t^{+}\right) & =\left(1-\mu_{3}\right) x_{3}(t), \quad t=(n+l-1) T, \\
x_{3}\left(t^{+}\right) & =x_{3}(t)+p, \quad t=n T, \\
x_{3}(t) & =x_{30} .
\end{aligned}
$$

System (10) is a periodically forced linear system easily used to obtain

$\left.\left.\left.\mu_{3}\right) \exp \left(-a_{3} T\right)\right)\right)$ is a positive periodic solution of system (10). Since the solution of (10) with initial value $x_{30} \geq 0$ is $\left(n \in N, x_{3}^{*}\left(0^{+}\right)=x_{3}^{*}\left(n T^{+}\right)=p /\left(1-\left(1-\mu_{3}\right) \exp \left(-a_{3} T\right)\right)\right.$, $x_{3}^{*}\left((n+l-1) T^{+}\right)=x_{3}^{*}\left(l T^{+}\right)=p\left(1-\mu_{3}\right) \exp \left(-a_{3} T\right) /(1-(1-$

$$
x_{3}(t)= \begin{cases}\left(1-\mu_{3}\right)^{n-1}\left(x_{3}\left(0^{+}\right)-\frac{p}{1-\left(1-\mu_{3}\right) \exp \left(-a_{3} T\right)} \exp \left(-a_{3} t\right)+x_{3}^{*}(t)\right), & (n-1) T<t \leq(n+l-1) T, \\ \left(1-\mu_{3}\right)^{n}\left(x_{3}\left(0^{+}\right)-\frac{p}{1-\left(1-\mu_{3}\right) \exp \left(-a_{3} T\right)} \exp \left(-a_{3} t\right)+x_{3}^{*}(t)\right), & (n+l-1) T<t \leq n T .\end{cases}
$$


Lemma 5 (see [18]). Suppose $x_{3}^{*}(t)$ is a positive periodic solution of (10) and $x_{30}>0$; then we get $\left|x_{3}(t)-x_{3}^{*}(t)\right| \rightarrow 0$ as $t \rightarrow+\infty$.

Lemma 6 (see [19]). Suppose $X(t)$ is a solution of (4) and $X\left(0^{+}\right) \geq 0$, and hence $X(t) \geq 0$ for all $t \geq 0$. It also has $X(t)>0(t \geq 0)$ if $X\left(0^{+}\right)>0$.

Lemma 7 (see [20]). Suppose function $u(t) \in P C^{1}\left(R_{+}, R\right)$ satisfies the following inequalities:

$$
\begin{aligned}
\frac{d u}{d t} & \leq f(t) u(t)+h(t), \quad t \neq \tau_{k}, t>0, \\
u\left(\tau_{k}\right) & \leq \alpha_{k} u\left(\tau_{k}\right)+\beta_{k}, \quad k \geq 0, \\
u\left(0^{+}\right) & \leq u_{0},
\end{aligned}
$$

where $f, h \in C\left(R_{+}, R\right)$ and $\alpha_{k} \geq 0, \beta_{k}$, and $u_{0}$ are constants $(k=1,2, \ldots)$. Then for $t>0$, one has

$$
\begin{aligned}
u(t) \leq & u_{0}\left(\prod_{0<\tau_{k}<t} \alpha_{k}\right) \exp \left(\int_{0}^{t} f(s) d s\right) \\
& +\int_{0}^{t}\left(\prod_{0 \leq \tau_{k}<t} \alpha_{k}\right) \exp \left(\int_{0}^{t} f(\gamma) d \gamma\right) h(d) d s \\
& +\sum_{0<\tau_{k}<t}\left(\prod_{\tau_{k}<\tau_{j}<t} \alpha_{j}\right) \exp \left(\int_{\tau_{k}}^{t} f(\gamma) d \gamma\right) \beta_{k} .
\end{aligned}
$$

\section{Main Theorems}

\subsection{Boundedness}

Theorem 8. For any solution $\left(x_{1}(t), x_{2}(t), x_{3}(t)\right)$ of system (4), there exists a constant $M>0$, such that $x_{1}(t) \leq M, x_{2}(t) \leq M$, and $x_{3}(t) \leq M$ hold for all t large enough.

Proof. Let $X(t)=\left(x_{1}(t), x_{2}(t), x_{3}(t)\right)$ be a solution of (4) with initial value $\left(x_{10}, x_{20}, x_{30}\right)$. $V_{0}$.

Define a function $\omega(t)=k_{1} x_{1}(t)+k_{2} x_{2}(t)+x_{3}(t), \omega(t) \epsilon$

When $t \neq(k+l-1) T, k T$, take a constant $L$ such that $0<L<a_{3}$; then by calculating the upper right derivative of $\omega(t, X(t))$ along the solution of system (4), we have

$$
\begin{aligned}
D^{+} \omega(t)+L \omega(t)= & k_{1}\left(a_{1}+L\right) x_{1}(t)-k_{1} b_{1} x_{1}^{2}(t) \\
& -k_{1} \alpha x_{1}(t) x_{2}(t) \\
& +k_{2}\left(a_{2}+L\right) x_{2}(t)-k_{2} b_{2} x_{2}^{2}(t) \\
& -k_{2} \beta x_{1}(t) x_{2}(t) \\
& +\left(L-a_{3}\right) x_{3}(t) \\
\leq & k_{1}\left(a_{1}+L\right) x_{1}(t)-k_{1} b_{1} x_{1}^{2}(t) \\
& +k_{2}\left(a_{2}+L\right) x_{2}(t)-k_{2} b_{2} x_{2}^{2}(t) \\
\leq & M_{0},
\end{aligned}
$$

where $M_{0}=k_{1}^{2}\left(a_{1}+L\right)^{2} / 4 k_{1} b_{1}+k_{2}^{2}\left(a_{2}+L\right)^{2} / 4 k_{2} b_{2}$.
Further, at moment $t=(k+l-1) T, \omega\left((k+l-1) T^{+}\right) \leq$ $\omega((k+l-1) T)$ and at $t=k T, \omega\left(k T^{+}\right) \leq \omega(k T)+p$. Then by Lemma 7 , for all $t \geq 0$, we get

$$
\begin{aligned}
& \omega(t) \leq \omega(0) e^{-L t}+\frac{k_{0}}{L}\left(1-e^{-L t}\right)+p \frac{e^{-L(t-T)}}{1-e^{L T}} \\
&+p \frac{e^{L T}}{e^{L T}-1} \longrightarrow \frac{k_{0}}{L}+p \frac{e^{L T}}{e^{L T}-1} \\
&(\text { as } t \longrightarrow \infty)=M_{1} .
\end{aligned}
$$

Hence $\omega(t)$ is bounded for sufficiently large $t$. Let $M=$ $\min \left\{M_{0}, M_{1}\right\}$; then $x_{1}(t), x_{2}(t)$, and $x_{3}(t)$ are bounded by $M$ for sufficiently large $t$. This completes the proof.

\subsection{Stability of Prey-Eradication Periodic Solution}

Theorem 9. Suppose $\left(x_{1}(t), x_{2}(t), x_{3}(t)\right)$ is any solution of (4); then the prey-eradication periodic solution $\left(0,0, x_{3}^{*}(t)\right)$ is globally asymptotically stable provided that

$$
T<\min \left\{\frac{1}{a_{1}} \ln \frac{1}{1-\mu_{1}}, \frac{1}{a_{2}} \ln \frac{1}{1-\mu_{2}}\right\} .
$$

Proof. The local stability of periodic solution $\left(0,0, x_{3}^{*}(t)\right)$ can be determined by considering the behavior of small amplitude perturbations of the solution.

Define $x_{1}(t)=u_{1}(t), x_{2}(t)=u_{2}(t)$, and $x_{3}(t)=x_{3}^{*}(t)+$ $u_{3}(t)$, and we get

$$
\left(\begin{array}{l}
u_{1}(t) \\
u_{2}(t) \\
u_{3}(t)
\end{array}\right)=\Phi(t)\left(\begin{array}{l}
u_{1}(0) \\
u_{2}(0) \\
u_{3}(0)
\end{array}\right), \quad 0 \leq t<T,
$$

where $u_{i}(t)(i=1,2,3)$ is a small perturbation. When $t \neq n T$, and $t \neq(n+l-1) T$, (4) can be expanded in a Taylor series. Then, neglecting higher-order terms, the linearized equations read

$$
\begin{aligned}
& u_{1}^{\prime}(t)=a_{1} u_{1}(t), \\
& u_{2}^{\prime}(t)=a_{2} u_{2}(t), \\
& u_{3}^{\prime}(t)=-a_{3} u_{3}(t) .
\end{aligned}
$$

Let $\Phi(t)$ be the fundamental matrix of above differential equations; then $\Phi(t)$ satisfies

$$
\begin{gathered}
\frac{d \Phi(t)}{d t}=\left(\begin{array}{ccc}
a_{1} & 0 & 0 \\
0 & a_{2} & 0 \\
0 & 0 & -a_{3}
\end{array}\right) \Phi(t), \\
\Phi(t)=\left(\begin{array}{ccc}
e^{a_{1}{ }^{t}} & 0 & 0 \\
0 & e^{a_{2}{ }^{t}} & 0 \\
0 & 0 & e^{-a_{3}{ }^{t}}
\end{array}\right) ;
\end{gathered}
$$


$\Phi(0)=I$ is the identity matrix. Then the linearization of system (4) becomes

$$
\begin{aligned}
& \left(\begin{array}{l}
u_{1}\left((n+l-1) T^{+}\right) \\
u_{2}\left((n+l-1) T^{+}\right) \\
u_{3}\left((n+l-1) T^{+}\right)
\end{array}\right) \\
& =\left(\begin{array}{ccc}
1-\mu_{1} & 0 & 0 \\
0 & 1-\mu_{2} & 0 \\
0 & 0 & 1-\mu_{3}
\end{array}\right)\left(\begin{array}{l}
u_{1}(n+l-1) T \\
u_{2}(n+l-1) T \\
u_{3}(n+l-1) T
\end{array}\right), \\
& \left(\begin{array}{l}
u_{1}\left(n T^{+}\right) \\
u_{2}\left(n T^{+}\right) \\
u_{3}\left(n T^{+}\right)
\end{array}\right)=\left(\begin{array}{lll}
1 & 0 & 0 \\
0 & 1 & 0 \\
0 & 0 & 1
\end{array}\right)\left(\begin{array}{l}
u_{1}(n T) \\
u_{2}(n T) \\
u_{3}(n T)
\end{array}\right) .
\end{aligned}
$$

The stability of periodic solution $\left(0,0, x_{3}^{*}(t)\right)$ of $(4)$ is determined by the eigenvalues of the matrix $A$ :

$$
A=\left(\begin{array}{ccc}
1-\mu_{1} & 0 & 0 \\
0 & 1-\mu_{2} & 0 \\
0 & 0 & 1-\mu_{3}
\end{array}\right)\left(\begin{array}{lll}
1 & 0 & 0 \\
0 & 1 & 0 \\
0 & 0 & 1
\end{array}\right) \Phi(T)
$$

If each of these eigenvalues of matrix $A$ is less than one, then the periodic solution $\left(0,0, x_{3}^{*}(t)\right)$ is locally stable. The three eigenvalues of the matrix $A$ are

$$
\begin{aligned}
& \lambda_{1}=\left(1-\mu_{1}\right) \exp \left(\int_{0}^{T} a_{1} d t\right), \\
& \lambda_{2}=\left(1-\mu_{2}\right) \exp \left(\int_{0}^{T} a_{2} d t\right), \\
& \lambda_{3}=\left(1-\mu_{3}\right) \exp \left(-a_{3} T\right) .
\end{aligned}
$$

From Floquet theory of impulsive differential equation [18], if $\left|\lambda_{i}\right|<1(i=1,2,3)$, then $\left(0,0, x_{3}^{*}(t)\right)$ is locally asymptotically stable. Here $\lambda_{3}$ is already less than one, so we only need to calculate $\left|\lambda_{i}\right|<1(i=1,2)$.

Actually,

$$
\begin{aligned}
& \left(1-\mu_{i}\right) \exp \left(\int_{0}^{T}\left(a_{i}\right) d t\right)<1, \quad(i=1,2), \\
& \Longleftrightarrow \int_{0}^{T}\left(a_{i}\right) d t<\ln \frac{1}{1-\mu_{i}}, \quad(i=1,2), \\
& \Longleftrightarrow a_{i} T<\ln \frac{1}{1-\mu_{i}}, \quad(i=1,2), \\
& \Longleftrightarrow T<\frac{1}{a_{i}} \ln \frac{1}{1-\mu_{i}}, \quad(i=1,2) .
\end{aligned}
$$

Hence, if $T<\min \left\{\left(1 / a_{1}\right) \ln \left(1 /\left(1-\mu_{1}\right)\right),\left(1 / a_{2}\right) \ln \left(1 /\left(1-\mu_{2}\right)\right)\right\}$, then $\left(0,0, x_{3}^{*}(t)\right)$ is locally asymptotically stable.
Next, we prove the global attractivity of $\left(0,0, x_{3}^{*}(t)\right)$. Choose $\varepsilon>0$ such that

$$
\begin{aligned}
& \xi_{1} \triangleq\left(1-\mu_{1}\right) \exp \left(\int_{0}^{T}\left(a_{1}-b_{1}\left(x_{1}(t)-\varepsilon\right)\right) d t\right)<1 \\
& \xi_{2} \triangleq\left(1-\mu_{2}\right) \exp \left(\int_{0}^{T}\left(a_{2}-b_{2}\left(x_{2}(t)-\varepsilon\right)\right) d t\right)<1 .
\end{aligned}
$$

From (4), we get

$$
\begin{aligned}
& \frac{d x_{1}(t)}{d t} \leq\left(a_{1}-b_{1} x_{1}(t)\right) x_{1}(t), \quad t \neq(n+l-1) T, \\
& x_{1}\left(t^{+}\right)=\left(1-\mu_{1}\right) x_{1}(t), \quad t=(n+l-1) T .
\end{aligned}
$$

It follows from (26) that

$$
\begin{aligned}
& x_{1}((n+l) T) \leq x_{1}\left((n+l-1) T^{+}\right) \\
& \cdot \exp \left(\int_{0}^{T}\left(a_{1}-b_{1}\left(x_{1}(t)-\varepsilon\right)\right) d t\right) \\
& \quad=x_{1}((n+l-1) T)\left(1-\mu_{1}\right) \\
& \quad \cdot \exp \left(\int_{0}^{T}\left(a_{1}-b_{1}\left(x_{1}(t)-\varepsilon\right)\right) d t\right) \\
& \quad=x_{1}((n+l-1) T) \xi_{1} .
\end{aligned}
$$

Continuing the iteration technique, we can obtain $x_{1}((n+$ $l) T) \leq x_{1}(l T) \xi_{1}^{n}$ and $x_{1}(l T) \xi_{1}^{n} \rightarrow 0$ as $n \rightarrow \infty$. Hence, $x_{1}((n+l) T) \rightarrow 0$ as $n \rightarrow \infty$. Then, $x_{1}(t) \rightarrow 0$ as $n \rightarrow \infty$ since $0<x_{1}(t)<x_{1}((n+l-1) T)\left(1-\mu_{1}\right) \exp \left(a_{1} T\right)$ for $(n+l-1) T<t \leq(n+l) T$. By the same way, we can get $x_{2}(t) \rightarrow 0$, as $n \rightarrow \infty$.

Now we prove $x_{3}(t) \rightarrow x_{3}^{*}(t)$ as $t \rightarrow \infty$. For $\varepsilon>$ $0\left(c_{1} k_{1} \sqrt{\varepsilon} /\left(1+d_{1} \varepsilon\right)+c_{2} k_{2} \sqrt{\varepsilon} /\left(1+d_{2} \varepsilon\right)<a_{3}\right)$ sufficiently small, there exists $T^{\prime}>0$ such that $0<x_{1}(t)<\varepsilon$ and $0<x_{2}(t)<\varepsilon, t>T^{\prime}$. Without loss of generality, we assume that $0<x_{1}(t)<\varepsilon$ and $0<x_{2}(t)<\varepsilon$ for all $t \geq 0$. Then from system (4) we obtain

$$
\begin{aligned}
-a_{3} x_{3}(t) \leq & \frac{d x_{3}(t)}{d t} \\
\leq & -a_{3} x_{3}(t) \\
& +\left(\frac{c_{1} k_{1} \sqrt{\varepsilon}}{1+d_{1} \sqrt{\varepsilon}}+\frac{c_{2} k_{2} \sqrt{\varepsilon}}{1+d_{2} \sqrt{\varepsilon}}\right) x_{3}(t)^{m} .
\end{aligned}
$$

Since $0<m \leq 1$, from (28), we have

$$
\begin{aligned}
-a_{3} x_{3}(t) & \leq \frac{d x_{3}(t)}{d t} \\
& \leq\left(-a_{3}+\frac{c_{1} k_{1} \sqrt{\varepsilon}}{1+d_{1} \sqrt{\varepsilon}}+\frac{c_{2} k_{2} \sqrt{\varepsilon}}{1+d_{2} \sqrt{\varepsilon}}\right) x_{3}(t) .
\end{aligned}
$$


Consider the following two comparison systems:

$$
\begin{aligned}
& \frac{d v_{1}(t)}{d t}=-a_{3} v_{1}(t), \quad t \neq n T, \quad t \neq(n+l-1) T, \\
& v_{1}\left(t^{+}\right)=\left(1-\mu_{3}\right) v_{1}(t), \quad t=(n+l-1), \\
& v_{1}\left(t^{+}\right)=v_{1}(t)+p, \quad t=n T, \\
& v_{1}\left(0^{+}\right)=x_{3}\left(0^{+}\right),
\end{aligned}
$$

$$
\begin{aligned}
\frac{d v_{2}(t)}{d t} & =\left(-a_{3}+\frac{c_{1} k_{1} \sqrt{\varepsilon}}{1+d_{1} \sqrt{\varepsilon}}+\frac{c_{2} k_{2} \sqrt{\varepsilon}}{1+d_{2} \sqrt{\varepsilon}}\right) v_{2}(t), \\
& t \neq n T, t \neq(n+l-1) T, \\
v_{2}\left(t^{+}\right) & =\left(1-\mu_{3}\right) v_{2}(t), \quad t=(n+l-1), \\
v_{2}\left(t^{+}\right) & =v_{2}(t)+p, \quad t=n T, \\
v_{2}\left(0^{+}\right) & =x_{3}\left(0^{+}\right) .
\end{aligned}
$$

We can obtain that the periodic solution $v_{1}^{*}(t)=x_{3}^{*}(t)$, and periodic solution of (31) is

$$
\begin{aligned}
& v_{2}^{*}(t) \\
& \quad= \begin{cases}\frac{p \exp \left(\left(-a_{3}+c_{1} k_{1} \sqrt{\varepsilon} /\left(1+d_{1} \sqrt{\varepsilon}\right)+c_{2} k_{2} \sqrt{\varepsilon} /\left(1+d_{2} \sqrt{\varepsilon}\right)\right)(t-(n-1) T)\right)}{1-\left(1-\mu_{3}\right) \exp \left(\left(-a_{3}+c_{1} k_{1} \sqrt{\varepsilon} /\left(1+d_{1} \sqrt{\varepsilon}\right)+c_{2} k_{2} \sqrt{\varepsilon} /\left(1+d_{2} \sqrt{\varepsilon}\right)\right) T\right)} & (n-1) T<t \leq(n+l-1) T, \\
\frac{p\left(1-\mu_{3}\right) \exp \left(\left(-a_{3}+c_{1} k_{1} \sqrt{\varepsilon} /\left(1+d_{1} \sqrt{\varepsilon}\right)+c_{2} k_{2} \sqrt{\varepsilon} /\left(1+d_{2} \sqrt{\varepsilon}\right)\right)(t-(n-1) T)\right)}{1-\left(1-\mu_{3}\right) \exp \left(\left(-a_{3}+c_{1} k_{1} \sqrt{\varepsilon} /\left(1+d_{1} \sqrt{\varepsilon}\right)+c_{2} k_{2} \sqrt{\varepsilon} /\left(1+d_{2} \sqrt{\varepsilon}\right)\right) T\right)}, & (n+l-1) T<t \leq n T .\end{cases}
\end{aligned}
$$

For any solutions $v_{1}(t)$ and $v_{2}(t)$ of the above two systems, respectively, we have $v_{1}(t) \rightarrow v_{1}^{*}(t)=x_{3}^{*}(t)$, and $v_{2}(t) \rightarrow$ $v_{2}^{*}(t) \rightarrow x_{3}^{*}(t)$ as $\varepsilon \rightarrow 0$ and $t \rightarrow \infty$. From Lemmas 5 and 4 , we have $v_{1}(t) \leq x_{3}(t) \leq v_{2}(t)$. Then, for any $\varepsilon_{1}>0$, there exists constant $T_{1}>0$ such that $v_{1}^{*}(t)-\varepsilon_{1}<x_{3}(t)<v_{2}^{*}(t)+\varepsilon_{1}, t \geq$ $T_{1}$. Let $\varepsilon \rightarrow 0$, and we have $x_{3}^{*}(t)-\varepsilon_{1}<x_{3}(t)<x_{3}^{*}(t)+\varepsilon_{1}$ for $t$ large enough, which implies $x_{3}(t) \rightarrow x_{3}^{*}(t)$ as $t \rightarrow \infty$. This completes the proof.

\subsection{Permanence of System (4)}

Theorem 10. System (4) is permanent if conditions $\left(H_{1}\right)$ and $\left(H_{2}\right)$ hold:

$\left(\mathrm{H}_{1}\right) p^{m}<\min \left\{\left(B_{1} / c_{1} A_{1}\right)\left(\ln \left(1-\mu_{1}\right)+\left(a_{1}-\alpha a_{2} / b_{2}\right) T\right)\right.$, $\left.\left(B_{2} / c_{2} A_{2}\right)\left(\ln \left(1-\mu_{2}\right)+\left(a_{2}-\beta a_{1} / b_{1}\right) T\right)\right\}$,

$\left(\mathrm{H}_{2}\right) a_{3}\left(\sqrt{b_{2}}+d_{2} \sqrt{a_{2}}\right)>c_{2} k_{2} \sqrt{a_{2}}, a_{3}\left(\sqrt{b_{1}}+d_{1} \sqrt{a_{1}}\right)>$ $c_{1} k_{1} \sqrt{a_{1}}$, where

$$
\begin{aligned}
A_{1} & =\left(1-\exp \left(-\left(a_{3}-\frac{c_{2} k_{2} \sqrt{a_{2}}}{\sqrt{b_{2}}+d_{2} \sqrt{a_{2}}}\right) m l T\right)+(1\right. \\
& \left.-\mu_{3}\right)^{m}\left(\exp \left(-\left(a_{3}-\frac{c_{2} k_{2} \sqrt{a_{2}}}{\sqrt{b_{2}}+d_{1} \sqrt{a_{2}}}\right) m l T\right)\right. \\
& \left.\left.-\exp \left(-\left(a_{3}-\frac{c_{2} k_{2} \sqrt{a_{2}}}{\sqrt{b_{2}}+d_{1} \sqrt{a_{2}}}\right) m T\right)\right)\right), \\
A_{2} & =\left(1-\exp \left(-\left(a_{3}-\frac{c_{1} k_{1} \sqrt{a_{1}}}{\sqrt{b_{1}}+d_{1} \sqrt{a_{1}}}\right) m l T\right)+(1\right. \\
& \left.-\mu_{3}\right)^{m}\left(\exp \left(-\left(a_{3}-\frac{c_{1} k_{1} \sqrt{a_{1}}}{\sqrt{b_{1}}+d_{1} \sqrt{a_{1}}}\right) m l T\right)\right.
\end{aligned}
$$

$$
\begin{gathered}
\left.\left.-\exp \left(-\left(a_{3}-\frac{c_{1} k_{1} \sqrt{a_{1}}}{\sqrt{b_{1}}+d_{1} \sqrt{a_{1}}}\right) m T\right)\right)\right) \\
B_{1}=d_{1}\left(a_{3}-\frac{c_{2} k_{2} \sqrt{a_{2}}}{\sqrt{b_{2}}+d_{2} \sqrt{a_{2}}}\right) m\left(1-\left(1-\mu_{3}\right)\right. \\
\left.\cdot \exp \left(-\left(a_{3}-\frac{c_{2} k_{2} \sqrt{a_{2}}}{\sqrt{b_{2}}+d_{2} \sqrt{a_{2}}}\right) T\right)\right)^{m}, \\
B_{2}=d_{1}\left(a_{3}-\frac{c_{1} k_{1} \sqrt{a_{1}}}{\sqrt{b_{1}}+d_{1} \sqrt{a_{1}}}\right) m\left(1-\left(1-\mu_{3}\right)\right. \\
\left.\cdot \exp \left(-\left(a_{3}-\frac{c_{1} k_{1} \sqrt{a_{1}}}{\sqrt{b_{1}}+d_{1} \sqrt{a_{1}}}\right) T\right)\right)^{m} .
\end{gathered}
$$

Proof. Suppose $X(t)=\left(x_{1}(t), x_{2}(t), x_{3}(t)\right)$ is any solution of system (4) with $X(0)>0$. From Theorem 8 , we know that there exists constant $M>0$ such that $x_{1}(t)<M, x_{2}(t)<$ $M, x_{3}(t)<M$ with $t \geq 0$. Noticing that $d x_{3}(t) / d t \geq$ $-a_{3} x_{3}(t)$, we consider this impulsive differential equation:

$$
\begin{aligned}
v_{3}^{\prime}(t) & =-a_{3} v_{3}(t), \quad t \neq n T, \quad t \neq(n+l-1) T, \\
v_{3}\left(t^{+}\right) & =\left(1-\mu_{3}\right) v_{3}(t), \quad t=(n+l-1) T, \\
v_{3}\left(t^{+}\right) & =v_{3}(t)+p, \quad t=n T, \\
v_{3}\left(0^{+}\right) & =x_{3}\left(0^{+}\right)>0 .
\end{aligned}
$$

Periodic solution of (34) is $v_{3}^{*}=p\left(1-\mu_{3}\right) \exp \left(-a_{3} T\right) /(1-(1-$ $\left.\left.\mu_{3}\right) \exp \left(-a_{3} T\right)\right)$. By Lemmas 4 and 6 , we have $x_{3}(t) \geq v_{3}(t)>$ $x_{3}^{*}(t)-\varepsilon$ and $x_{3}(t) \geq p \exp \left(-r_{3}(t-n T)\right) /\left(1-\exp \left(-r_{3} T\right)\right)-$ $\varepsilon=m_{3}(\varepsilon>0)$ for $t$ sufficiently large. Now we want to find 
$\widetilde{m_{1}}>0, \widetilde{m_{2}}>0$ such that $x_{1}(t) \geq \widetilde{m_{1}}>0, x_{2} \geq \widetilde{m_{2}}>0$ for $t$ large enough. We prove it by the following two steps.

Step 1. We conclude that there exist $m_{1}>0, m_{2}>0, t_{1}, t_{2} \in$ $(0,+\infty)$ such that $x_{1}\left(t_{1}\right) \geq m_{1}, x_{2}\left(t_{2}\right) \geq m_{2}$. Otherwise there will be another three cases.

Case 1. There exists a constant $t_{2}>0$ such that $x_{2}\left(t_{2}\right) \geq m_{2}$, but $x_{1}(t)<m_{1}$ for all $t>0$.

Case 2. There exists a constant $t_{1}>0$ such that $x_{1}\left(t_{1}\right) \geq m_{1}$, but $x_{2}(t)<m_{2}$ for all $t>0$.

Case 3. Consider $x_{1}(t)<m_{1}$, and $x_{2}(t)<m_{2}$ for all $t>0$.

For Case 1 , choose a sufficiently small constant $\eta_{1}>0$ such that

$$
\begin{aligned}
\delta_{1} & =\left(1-\mu_{1}\right) \\
& \cdot \exp \left(\int_{(n+l-1) T}^{(n+l) T}\left(a_{1}-b_{1} M-\alpha M-c_{1} \frac{M}{d_{1}}\left(\gamma_{1}^{*}(t)+\eta_{1}\right)^{m}\right) d t\right) \\
& >1 .
\end{aligned}
$$

$$
\gamma_{1}^{*}(t)= \begin{cases}\frac{p \exp \left(F_{1}(t-(n-1) T)\right)}{1-\left(1-\mu_{3}\right) \exp \left(F_{1} T\right)}, & (n-1) T<t \leq(n+l-1) T, \\ \frac{p\left(1-\mu_{3}\right) \exp \left(F_{1}(t-(n-1) T)\right)}{1-\left(1-\mu_{3}\right) \exp \left(F_{1} T\right)}, & (n+l-1) T<t \leq n T .\end{cases}
$$

$$
\begin{aligned}
& x_{3}^{\prime}=x_{3}\left(\frac{k_{1} c_{1} \sqrt{x_{1}} x_{3}{ }^{m-1}}{1+d_{1} \sqrt{x_{1}(t)}}+\frac{k_{2} c_{2} \sqrt{x_{2}} x_{3}{ }^{m-1}}{1+d_{2} \sqrt{x_{2}(t)}}-a_{3}\right) \\
& \quad \leq x_{3}\left(k_{1} c_{1}\left(m_{3}\right)^{m-1} \sqrt{M}+k_{2} c_{2}\left(m_{3}\right)^{m-1} \sqrt{M}-a_{3}\right) \\
& \quad \triangleq F_{1} x_{3}(t), \quad t \neq n T, \quad t \neq(n+l-1) T, \\
& x_{3}\left(t^{+}\right)=\left(1-\mu_{3}\right) x_{3}(t), \quad t=(n+l-1) T, \\
& x_{3}\left(t^{+}\right)=x_{3}(t)+p, \quad t=n T .
\end{aligned}
$$

For any solution $\gamma_{1}(t)$ of (37), we have $\gamma_{1}(t) \rightarrow \gamma_{1}^{*}(t)$. By comparison theorem, we can easily obtain $x_{3}(t) \leq \gamma_{1}(t)$ as $t \rightarrow+\infty$. Hence, there exists $T_{1}>0$, when $t>T_{1}, x_{3}(t) \leq$ $\gamma_{1}(t)<\gamma_{1}^{*}(t)+\eta_{1}$.

Then we have

$$
\begin{aligned}
& x_{1}^{\prime}(t) \\
& \geq x_{1}(t)\left(a_{1}-b_{1} M-\alpha M-c_{1} \frac{M}{d_{1}}\left(\gamma_{1}^{*}(t)+\eta_{1}\right)^{m}\right), \\
& t \neq n T, t \neq(n+l-1) T, \\
& x_{1}\left(t^{+}\right)=\left(1-\mu_{1}\right) x_{1}(t), \quad t=(n+l-1) T, \\
& x_{1}\left(t^{+}\right)=0, \quad t=n T ;
\end{aligned}
$$

$N_{1} \in N$ and $\left(N_{1}+l-1\right) T \geq T_{1}$. Integrating (39) on $((n+l-$ 1)T, $(n+l) T], n \geq N_{1}$, we get

$$
\begin{aligned}
& x_{1}((n+l) T) \geq x_{1}\left((n+l-1) T^{+}\right) \\
& \quad \cdot \exp \left(\int_{(n+l-1) T}^{(n+l) T}\left(a_{1}-b_{1} M-\alpha M-\frac{c_{1}}{d_{1}} M\left(\gamma_{1}^{*}(t)+\eta_{1}\right)^{m}\right) d t\right) \\
& \quad=x_{1}((n+l-1) T)\left(1-\mu_{1}\right)
\end{aligned}
$$

Then we have

Consider the following comparison equation:

$$
\begin{aligned}
\gamma_{1}^{\prime} & =F_{1} \gamma_{1}, \quad t \neq n T, \quad t \neq(n+l-1) T, \\
\gamma_{1}\left(t^{+}\right) & =\left(1-\mu_{3}\right) \gamma_{1}, \quad t=(n+l-1) T, \\
\gamma_{1}\left(t^{+}\right) & =\gamma+p, \quad t=n T, \\
\gamma_{1}\left(0^{+}\right) & =x_{3}\left(0^{+}\right)>0 ;
\end{aligned}
$$

the periodic solution of (37) reads

$$
\begin{aligned}
& \cdot \exp \left(\int_{(n+l-1) T}^{(n+l) T}\left(a_{1}-b_{1} M-\alpha M-\frac{c_{1}}{d_{1}} M\left(\gamma_{1}^{*}(t)+\eta_{1}\right)^{m}\right) d t\right) \\
& =x_{1}((n+l-1) T) \delta_{1} .
\end{aligned}
$$

Continuing the iteration technique, we can obtain $x_{1}\left(\left(N_{1}+\right.\right.$ $k) T) \geq x_{1}\left(\left(N_{1}\right) T\right) \sigma_{1}^{k} \rightarrow \infty$ as $k \rightarrow \infty$, which is a contradiction. Similar to Case 1 , we can prove Case 2 is contradictory.

Next we consider Case 3. Using the assumption in Case 3, it is easy to get

$$
\begin{aligned}
& x_{3}^{\prime}=x_{3}\left(\frac{k_{1} c_{1} \sqrt{x_{1}} x_{3}{ }^{m-1}}{1+d_{1} \sqrt{x_{1}(t)}}+\frac{k_{2} c_{2} \sqrt{x_{2}} x_{3}{ }^{m-1}}{1+d_{2} \sqrt{x_{2}(t)}}-a_{3}\right) \\
& \quad \leq x_{3}\left(k_{1} c_{1}(M)^{m-1} \sqrt{m_{1}}+k_{2} c_{2}(M)^{m-1} \sqrt{m_{2}}-a_{3}\right) \\
& \quad \triangleq x_{3}(t) F_{2}, \quad t \neq n T, \quad t \neq(n+l-1) T, \\
& x_{3}\left(t^{+}\right)=\left(1-\mu_{3}\right) x_{3}(t), \quad t=(n+l-1) T, \\
& x_{3}\left(t^{+}\right)=x_{3}(t)+p, \quad t=n T .
\end{aligned}
$$



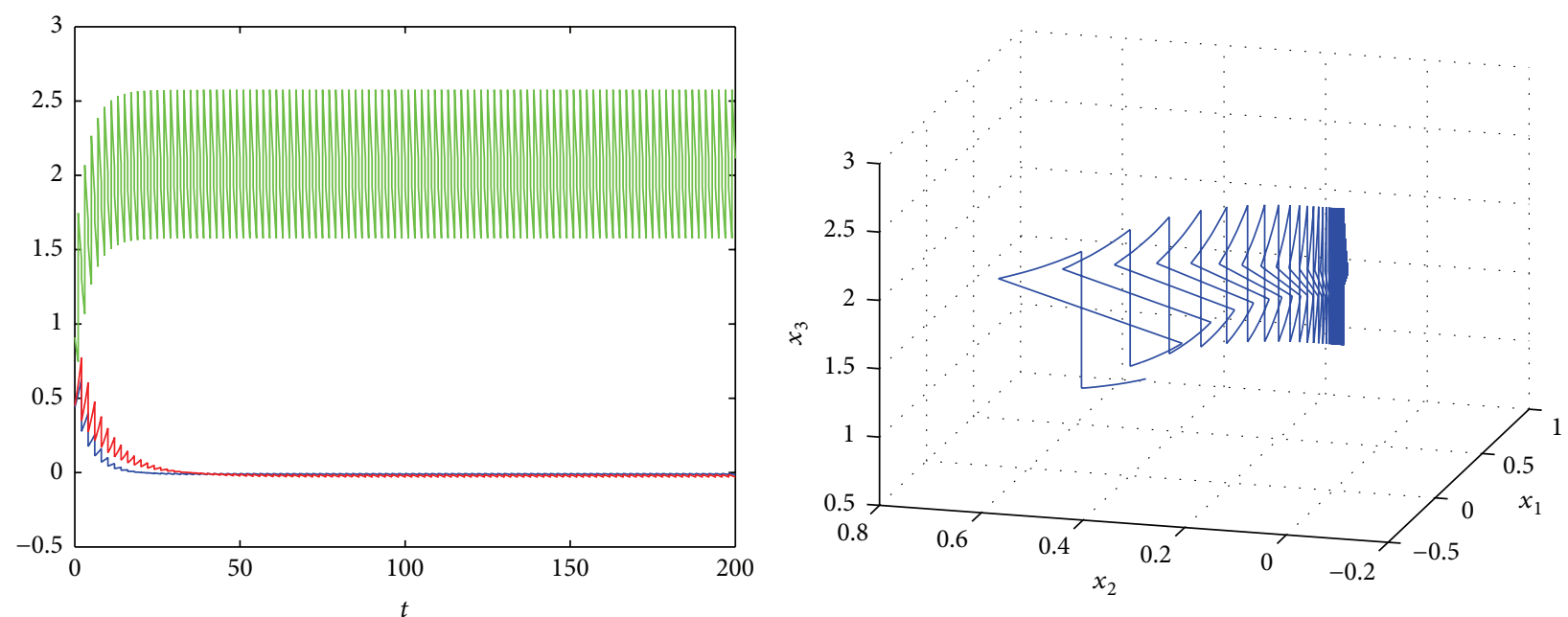

$-x_{1}$
$-x_{2}$
$-x_{3}$

(a)

(b)
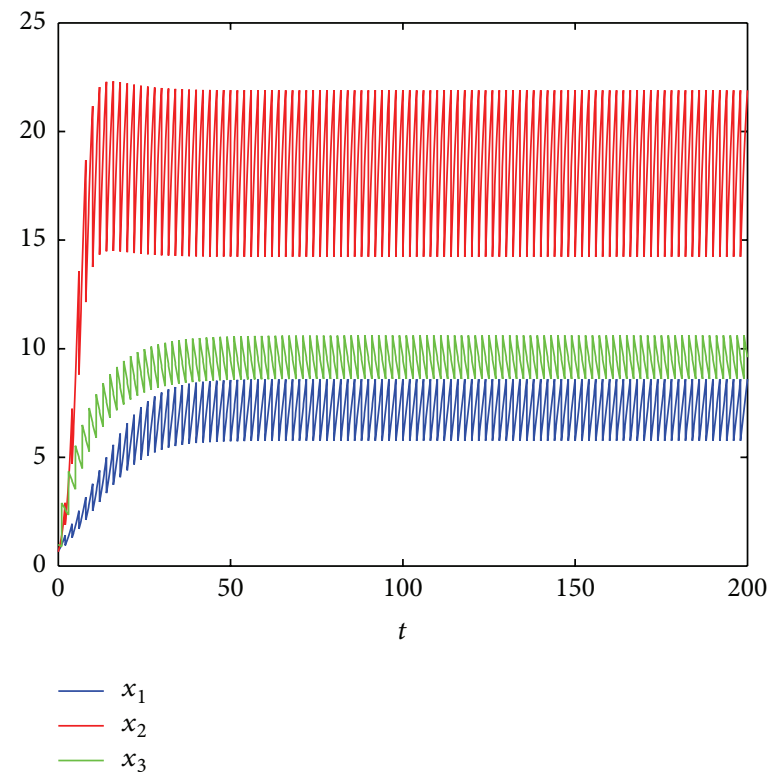

(c)

FIGURE 1: A prey-eradication periodic solution with initial values of $x_{1}(0)=2, x_{2}(0)=2$, and $x_{3}(0)=3$; (a) time series of $x_{1}(t), x_{2}(t), x_{3}(t)$, which are simulated 100 cycles in the interval [0,200]; (b) phase portrait of $x_{1}(t), x_{2}(t), x_{3}(t)$ : the solution goes gradually into the stable state from the initial point $[2,2,3]$; (c) the dynamic behaviors of system (4) with mutual interference of predator $m$ as $m=0.6$.

By the same discussion as Case 1, we can find a contradiction. Hence, from the above three cases, there exist $m_{1}>0$ and $m_{2}>0, t_{1}, t_{2} \in(0,+\infty)$ such that $x_{1}\left(t_{1}\right) \geq m_{1}, x_{2}\left(t_{2}\right) \geq$ $m_{2}$.

Step 2. If $x_{1}(t) \geq m_{1}$ for all $t \geq t_{1}$, then our aim is achieved. Otherwise, if $x_{1}(t)<m_{1}$ for some $t \geq t_{1}$, then we only need to consider those solutions which leave the region $\left\{X(t) \in R_{+}^{3}\right.$ : $\left.x_{1}(t) \geq m_{1}\right\}$ and reenter it again. Let $t^{*}=\inf _{t \geq t_{1}}\left\{x_{1}(t)<m_{1}\right\}$, and then there are two possible cases for $t^{*}$.
Case 1. If $t^{*}=\left(n_{1}+l-1\right) T, n_{1} \in N$, then $x_{1}(t) \geq m_{1}$ for $t \in\left[t_{1}, t^{*}\right)$ and $\left(1-\mu_{1}\right) m_{1} \leq x_{1}\left(t^{*+}\right)=\left(1-\mu_{1}\right) x_{1}\left(t^{*}\right)<m_{1}$. Choose $n_{2}, n_{3} \in N$ such that

$$
\begin{aligned}
& \left(n_{2}-1\right) T>\frac{\ln \left(\eta_{1} /(M+p)\right)}{F_{1}}, \\
& \left(1-\mu_{1}\right)^{n_{2}} \delta_{1}^{n_{3}} \exp \left(\delta n_{2} T\right) \\
& \quad>\left(1-\mu_{1}\right)^{n_{2}} \delta_{1}^{n_{3}}\left(\delta\left(n_{2}+1\right) T\right)>1,
\end{aligned}
$$




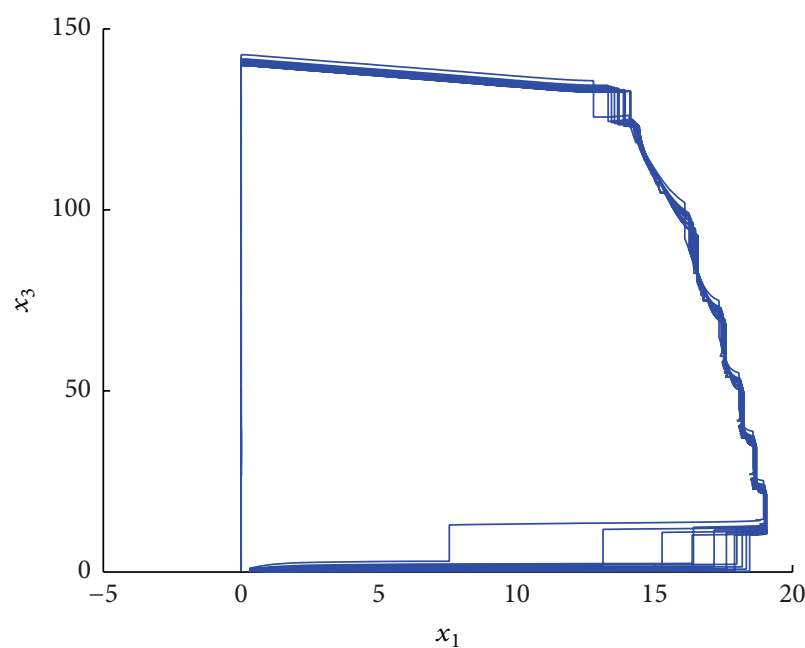

(a)

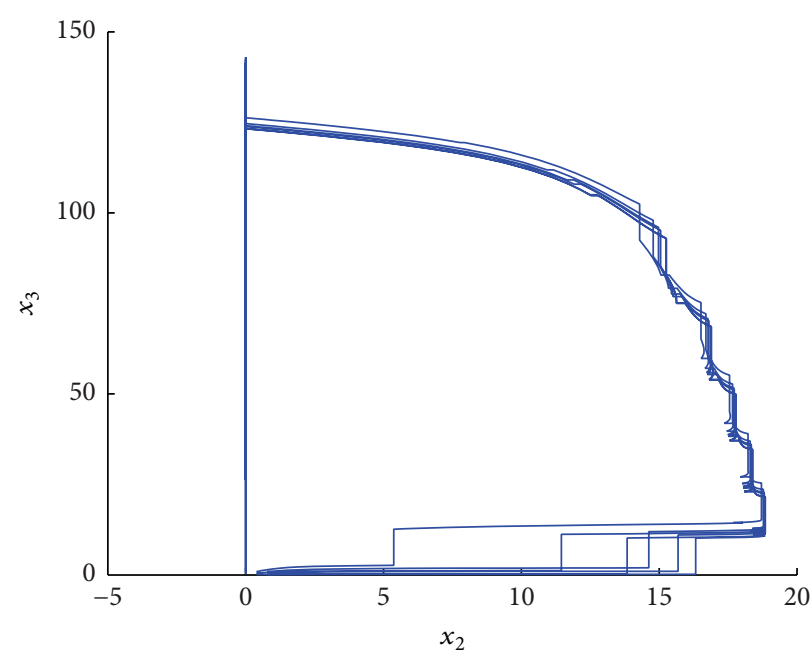

(b)

Figure 2: Dynamic behavior of the prey-eradication periodic solution with different initial values and the parameters are same as Figure 1. (a) Phase portrait of $x_{1}(t)$ and $x_{3}(t)$ with different initial values. (b) Phase portrait of $x_{2}(t)$ and $x_{3}(t)$ with different initial values.

where $\delta=a_{1}-b_{1} M-\alpha M-c_{1} M^{m}\left(M / d_{1}\right)<0$. Let $\bar{T}=$ $n_{2} T+n_{3} T$. We claim there must be $t^{\prime} \in\left(t^{*}, t^{*}+\bar{T}\right)$ such that $x_{1}\left(t^{\prime}\right)>m_{1}$. Otherwise we have $x_{1}\left(t^{\prime}\right)<m_{1}$, considering (37) with $\gamma_{1}\left(n_{1} T^{+}\right)=x_{3}\left(n_{1} T^{+}\right)$, and then we get

$$
\begin{aligned}
& \gamma_{1}(t) \\
& = \begin{cases}\left(1-\mu_{3}\right)^{n-\left(n_{1}+l\right)}\left(x_{3}\left(n_{1} T^{+}\right)-\frac{p}{1-\left(1-\mu_{3}\right) \exp \left(F_{1} T\right)}\right) \exp \left(F_{1}\left(t-n_{1} T\right)\right)+\gamma_{1}^{*}(t), & (n-1) T<t \leq(n+l-1) T, \\
\left(1-\mu_{3}\right)^{n-\left(n_{1}+l-1\right)}\left(x_{3}\left(n_{1} T^{+}\right)-\frac{p}{1-\left(1-\mu_{3}\right) \exp \left(F_{1} T\right)}\right) \exp \left(F_{1}\left(t-n_{1} T\right)\right)+\gamma_{1}^{*}(t), & (n+l-1) T<t \leq n T,\end{cases}
\end{aligned}
$$

and $n_{1}+1 \leq n \leq n_{1}+n_{2}+n_{3}+1$. Then $\left|\gamma_{1}(t)-\gamma_{1}^{*}(t)\right|<$ $(M+p) \exp \left(F_{1}\left(t-n_{1} T\right)\right)<\eta_{1}$ and $x_{3}(t) \leq \gamma_{1}(t)<\gamma_{1}^{*}(t)+\eta_{1}$ for $t^{*}+n_{2} T \leq t \leq t^{*}+\bar{T}$. These imply that (39) holds for $t^{*}+n_{2} T \leq t \leq t^{*}+\bar{T}$. By the same discussion of Step 1, we obtain $x_{1}\left(t^{*}+\bar{T}\right) \geq x_{1}\left(t^{*}+n_{2} T\right) \delta_{1}^{n_{3}}$.

From the first equation of (4), we have

$$
\begin{aligned}
x_{1}^{\prime}(t) & \geq x_{1}(t)\left(a_{1}-b_{1} M-\alpha M-c_{1} M^{m} \frac{M}{d_{1}}\right) \\
& =\delta x_{1}(t), \quad t \neq n T, \\
x_{1}\left(t^{+}\right) & =\left(1-\mu_{1}\right) x_{1}(t), \quad t=n T .
\end{aligned}
$$

Integrating (44) on $t \in\left[t^{*}, t^{*}+n_{2} T\right]$, then $x_{1}\left(t^{*}+\right.$ $\left.n_{2} T\right) \geq m_{1}\left(1-\mu_{1}\right)^{n_{2}} \exp \left(\delta n_{2} T\right)$ and $x_{1}\left(t^{*}+\bar{T}\right) \geq m_{1}(1-$ $\left.\mu_{1}\right)^{n_{2}} \delta_{1}^{n_{3}} \exp \left(\delta n_{2} T\right)>m_{1}$, which is a contradiction with $x_{1}(t)<m_{1}$. Hence, $x_{1}(t)>m_{1}$ holds for $t \in\left(t_{1}, t^{*}\right)$. Let $\bar{t}=\inf _{t \geq t^{*}}\left\{x_{1}(t) \geq m_{1}\right\}$, and then $x_{1}(t) \leq m_{1}, x_{1}(\bar{t})=m_{1}$ and $x_{1}(t) \geq m_{1}\left(1-\mu_{1}\right)^{n_{2}+n_{3}} \exp \left(\left(n_{2}+n_{3}\right) \delta T\right) \triangleq m_{1}^{\prime}$ for $t^{*}<t<\bar{t}$. So $x_{1}(t) \geq m_{1}^{\prime}$ holds for $t^{*}<t<\bar{t}$. If $t>\bar{t}$, the same arguments can be expanded since $x_{1}(\bar{t}) \geq m_{1}$.

Case 2. If $t^{*} \neq\left(n_{1}+l-1\right) T, n_{1} \in N$, then $x_{1}(t) \geq m_{1}$ for $t_{1} \leq t<t^{*}$ and $x_{1}\left(t^{*}\right)=m_{1}$.

Suppose $t^{*} \in\left(\left(n_{1}^{\prime}+l-1\right) T,\left(n_{1}^{\prime}+l\right) T\right), n_{1}^{\prime} \in N$, and then there are two possible cases for $t \in\left(t^{*},\left(n_{1}^{\prime}+l\right) T\right)$.

Case 2.1. $x_{1}(t) \leq m_{1}$ holds for all $t \in\left(t^{*},\left(n_{1}^{\prime}+l\right) T\right)$. Similar to Case 1 , we can prove that there must exist $t_{1}^{\prime} \in\left[\left(n_{1}^{\prime}+l\right) T,\left(n_{1}^{\prime}+\right.\right.$ l) $T+\bar{T}]$ such that $x_{1}\left(t_{1}^{\prime}\right)>m_{1}$; we omit it here.

Let $\widetilde{t}=\inf _{t \geq t^{*}}\left\{x_{1}(t)>m_{1}\right\}$, and then $x_{1}(t) \leq m_{1}$ holds for $t^{*} \leq t<\tilde{t}$. Then we have $x_{1}(t) \geq m_{1}\left(1-\mu_{1}\right)^{n_{2}+n_{3}+1} \exp \left(\left(n_{2}+\right.\right.$ $\left.\left.n_{3}\right) \delta T\right) \triangleq \widetilde{m_{1}}$, and $x_{1}(t) \geq \widetilde{m_{1}}$ for $t \in\left(t^{*}, \widetilde{t}\right)$. For $t>\widetilde{t}$, the same arguments can be followed since $x_{1}(\widetilde{t}) \geq \widetilde{m_{1}}$. 


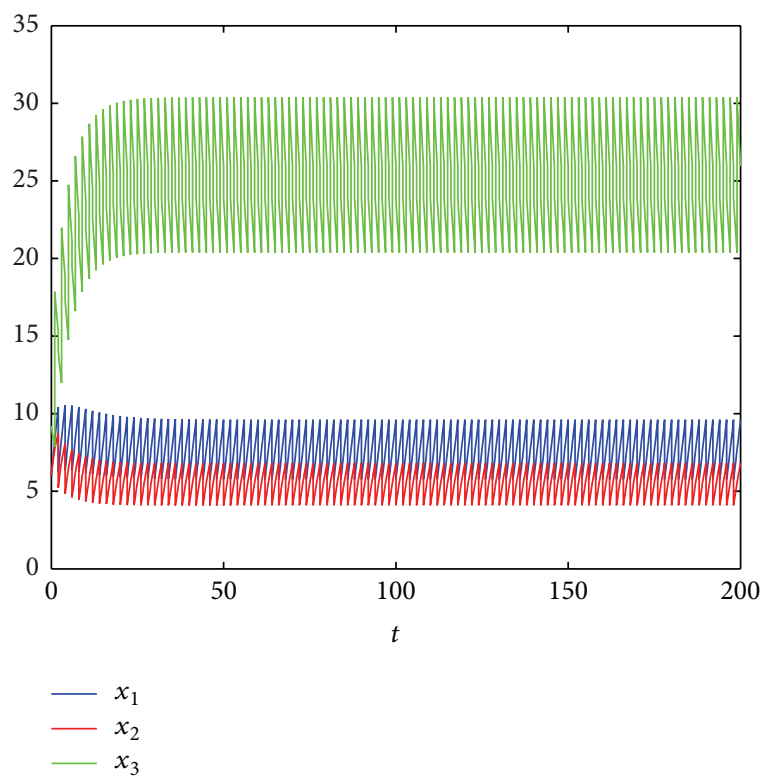

(a)

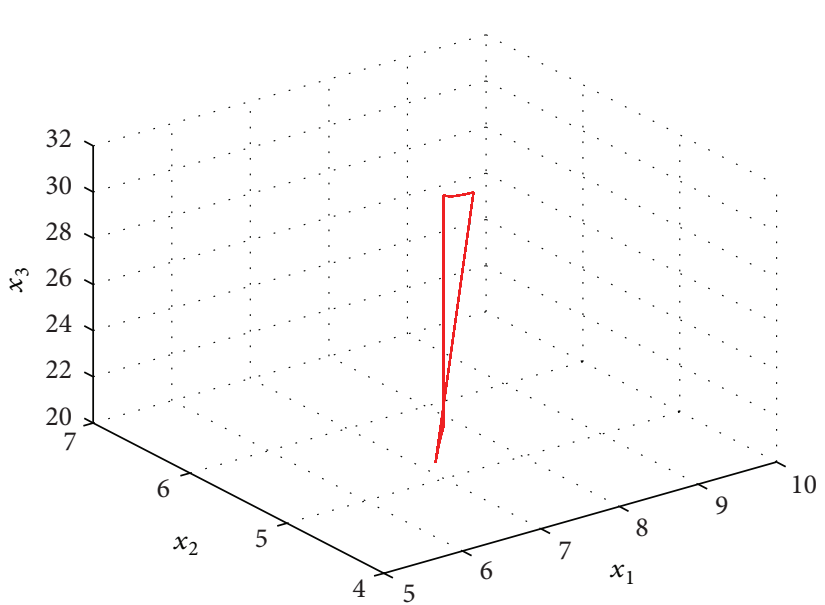

(b)

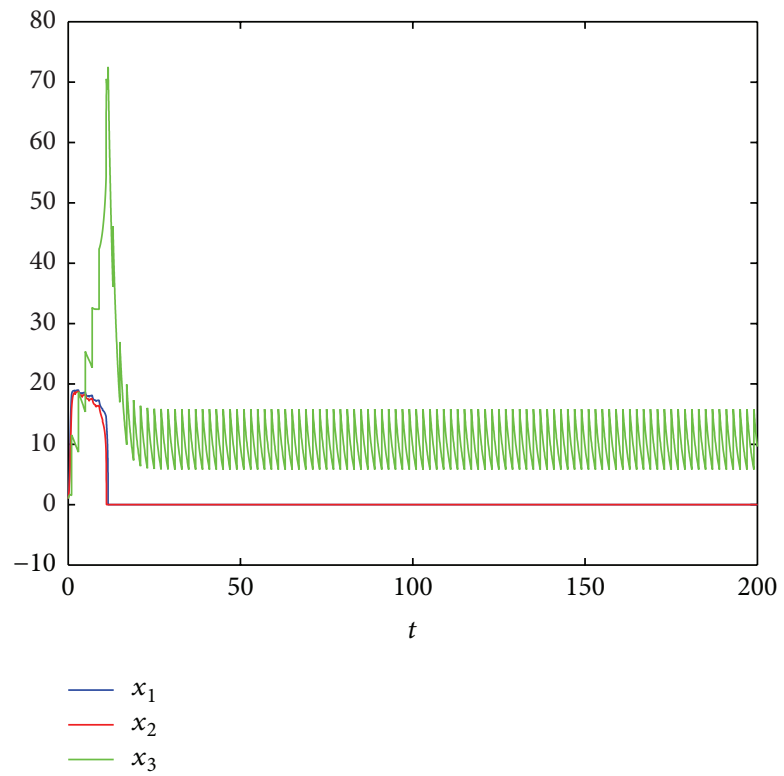

(c)

FIGURE 3: (a) is the existence of the positive periodic solution of system (4) with initial values of $x_{1}(t)=2, x_{2}(t)=2, x_{3}(t)=3$, which implies that system (4) is permanent; (b) is phase portrait of positive periodic solution of system (4); (c) is dynamic behavior of system (4) with the mutual interference of the predator $m=0.66$; other parameters are also the same as (a).

Case 2.2. There exist $t^{*}<t<\left(n_{1}^{\prime}+l\right) T$ such that $x_{1}(t)>m_{1}$. Let $\bar{t}=\inf _{t \geq t^{*}}\left\{x_{1}(t)>m_{1}\right\}$, and then $x_{1}(t) \leq m_{1}$ for $t \in\left(t^{*}, \bar{t}\right)$ and $x_{1}(t)=m_{1}$. When $t \in\left(t^{*}, \hat{t}\right),(44)$ holds. Integrating (44) on $\left(t^{*}, \bar{t}\right)$, then $x_{1}(t) \geq x_{1}\left(t^{*}\right) \exp \left(\delta\left(t-t^{*}\right)\right) \geq \widetilde{m_{1}} \exp (\delta T)>$ $m_{1}$. Since $x_{1}(t) \geq \widetilde{m_{1}}$ for $t>t^{*}$, the same arguments can be used; then $x_{1}(t) \geq \widetilde{m_{1}}$ for all $t \geq t_{1}$.

Thus in both cases, we conclude that $x_{1}(t) \geq \widetilde{m_{1}}$ holds for all $t \geq t_{1}$. Similarly, we have $x_{2}(t) \geq \widetilde{m_{2}}$ for all $t \geq t_{2}$. Let $m_{4}=$ $\max \left\{\widetilde{m_{1}}, \widetilde{m_{2}}, m_{3}\right\}$, and then we have $m_{4}<x_{1}, x_{2}, x_{3}<M$. The proof is completed.

\section{Single Chemical Control}

If $p=0$, system (4) concerns the single chemical control. Then we have the following corollary.

Corollary 11. Assume $p=0$ and $l=1$.

(i) Let $\left(x_{1}(t), x_{2}(t), x_{3}(t)\right)$ be any solution of (4). Then the solution $\left(0,0, x_{3}^{*}(t)\right)$ is locally stable if

$$
T<\min \left\{\frac{1}{a_{1}} \ln \frac{1}{1-\mu_{1}}, \frac{1}{a_{2}} \ln \frac{1}{1-\mu_{2}}\right\} .
$$




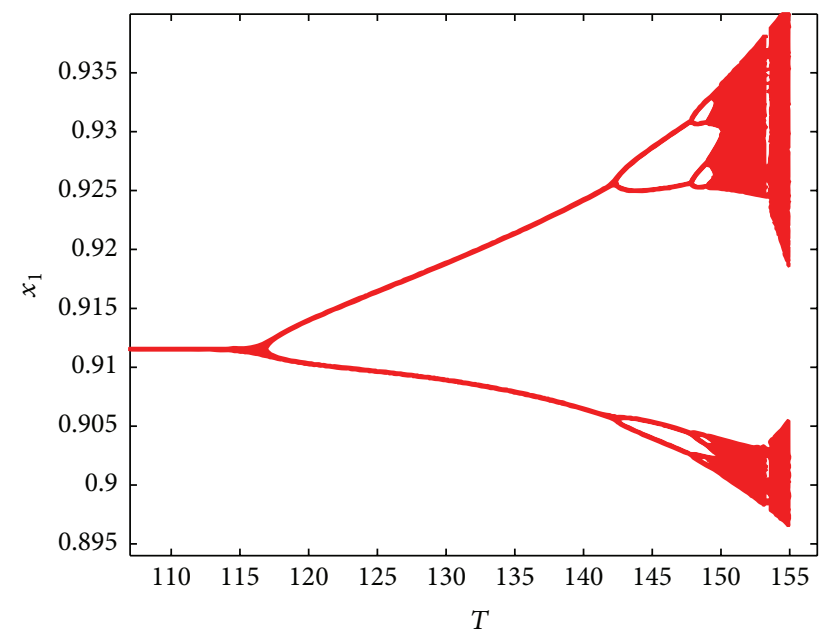

(a)

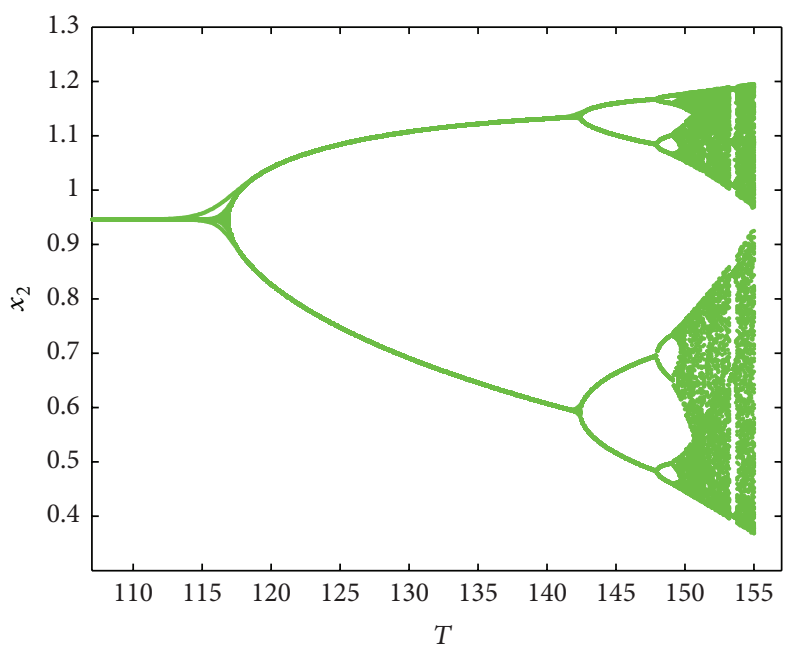

(b)

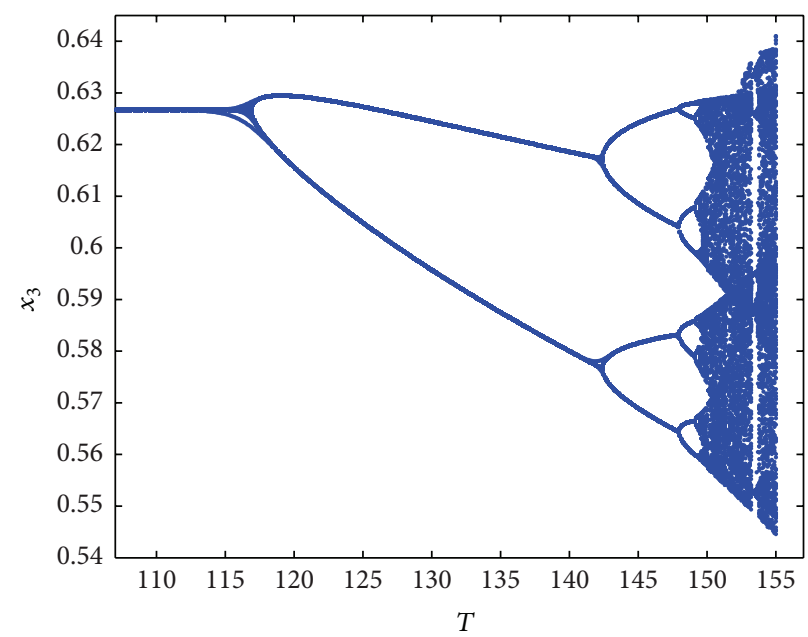

(c)

FIGURE 4: Bifurcation diagrams of system (4) with $x_{1}(0)=0.7, x_{2}(0)=0.8, x_{3}(0)=0.5$. The system exhibits stable solution, periodic doubling bifurcation, and chaotic phenomenon corresponding with different $T$. (a) is the bifurcation diagram of population $x_{1}(t)$; (b) is the bifurcation diagram of population $x_{2}(t)$; (c) is the bifurcation diagram of population $x_{3}(t)$.

(ii) System (4) is permanent if $a_{3}\left(b_{2} d_{1}+a_{2} d_{3}\right) \quad>$ $c_{2} k_{2} a_{2}, a_{3}\left(b_{1} d_{1}+a_{1} d_{2}\right)>c_{1} k_{1} a_{1}$ and

$$
\begin{aligned}
T & >\max \left\{\frac{1}{a_{1}-\alpha a_{2} / b_{2}} \ln \frac{1}{1-\mu_{1}}, \frac{1}{a_{2}-\beta a_{1} / b_{1}}\right. \\
& \left.\cdot \ln \frac{1}{1-\mu_{2}}\right\} .
\end{aligned}
$$

\section{Examples and Simulations}

In this section, we give some examples and numerical simulations to verify our theoretical results and further study the complexity and variety of system (4).

Firstly, let $a_{1}=0.2, a_{2}=0.3, a_{3}=0.2, b_{1}=0.03, b_{2}=0.04, c_{1}$ $=0.1, c_{2}=0.1, d_{1}=100, d_{2}=100, \mu_{1}=0.56, \mu_{2}=0.55, \mu_{3}=0.09$, $\alpha=0.003, \beta=0.004, k_{1}=0.75, k_{2}=0.75, m=1, l=0.2, T=10$, and $p=1$. By verification, these parameters satisfy conditions of Theorem 9; then the prey-eradication periodic solution is global asymptotic stability. With Matlab, by simulation, the asymptotic stability can be illustrated by Figures 1(a), 1(b), and 2. Figures 1(a) and 1(b) show the existence of the preyeradication periodic solution. Figure 2 shows the attractivity of prey-eradication periodic solution. If we change the mutual interference of the predator $m$ and other parameters are the same as Figure 1(a), then we get another dynamical portrait that is very different from Figure 1(a). We give an example for $m=0.6$ (see Figure 1(c)), which shows that the mutual interference of the predator $m$ affects the dynamical behaviors of system (4) to be more complex.

Secondly, let $x_{1}(t)=2, x_{2}(t)=2, x_{3}(t)=3, a_{1}=0.6$, $a_{2}=0.8, a_{3}=0.2, b_{1}=0.03, b_{2}=0.07, c_{1}=0.8, c_{2}=$ $0.8, d_{1}=30, d_{2}=30, \mu_{1}=0.4, \mu_{2}=0.4, \mu_{3}=0.08, \alpha$ $=0.005, \beta=0.005, k_{1}=0.8, k_{2}=0.8, m=1, l=1, T$ $=10$, and $p=10$. By verification, these parameters satisfy conditions of Theorem 10. Then we conclude that system 


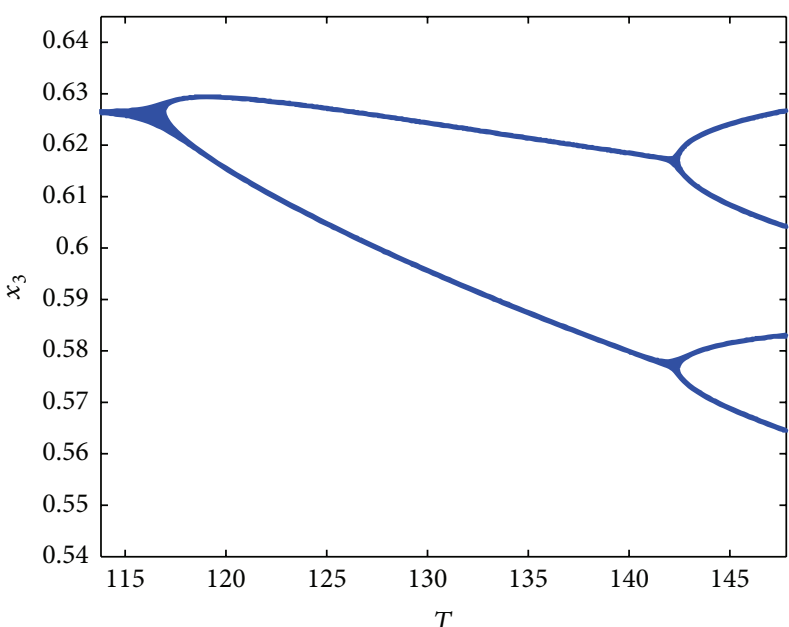

(a)

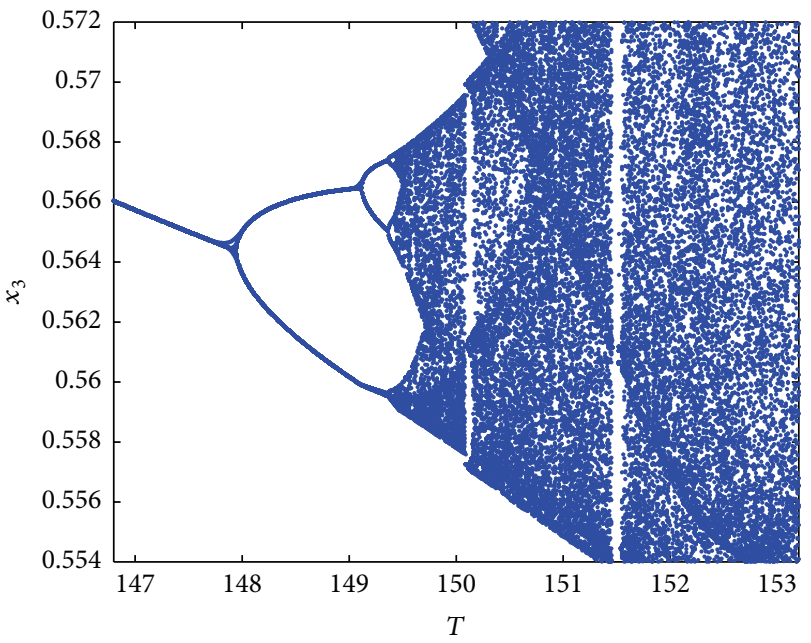

(c)

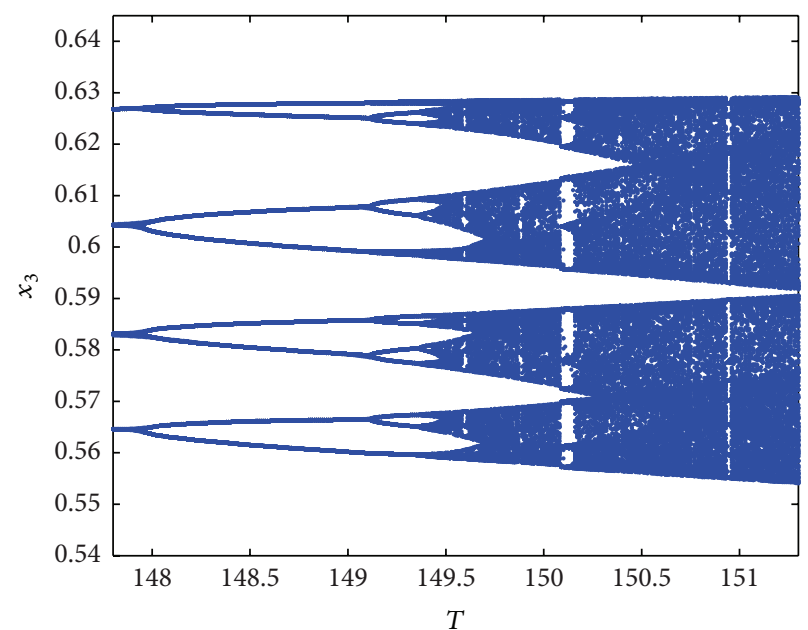

(b)

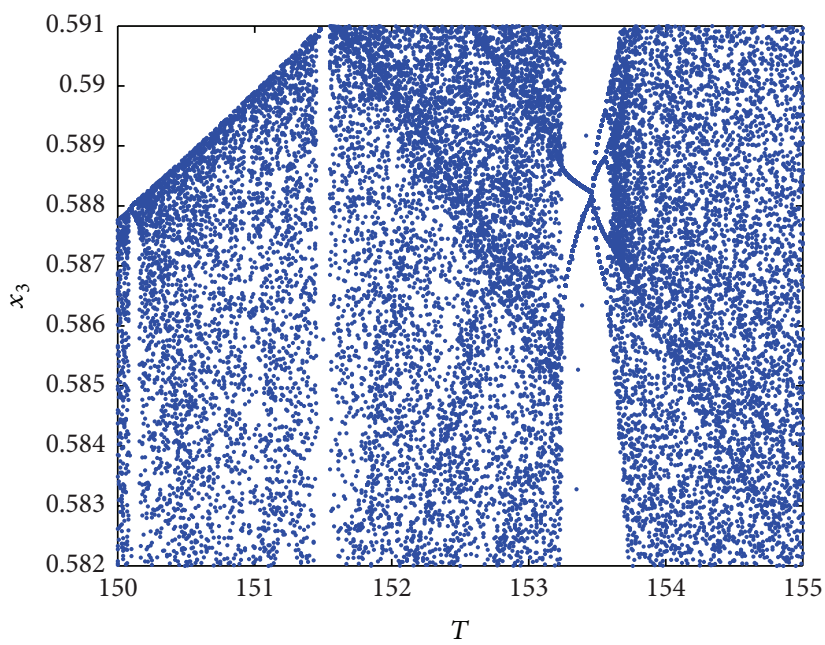

(d)

Figure 5: The magnified parts of Figure 4(c) of dynamic behavior of $x_{3}$. (a) and (b) are period solutions. (c) is a typical chaotic oscillation when $T=151.6$. (d) is the symmetry-breaking bifurcations when $T=153.5$.

(4) is permanent from Theorem 10. By simulation, we can verify the same results. Figure $3(\mathrm{a})$ is the time series of the three species in the interval $[0,200]$. In addition, we give the phase portrait of a positive periodic solution of system (4) in Figure 3(b). Similarly, if we change the mutual interference of the predator $m$ and other parameters are same as Figure 3(a), then we get another dynamical picture that is very different from Figure 3(a). We give an example for $m=0.66$ (see Figure 3(c)). It also shows that the parameter $m$ affects the dynamical behaviors of system (4).

Thirdly, by numerical analysis, we aim to investigate the bifurcation diagrams of impulsive period $T$. Let $x_{1}(0)=2$, $x_{2}(0)=2$, and $x_{3}(0)=3$, and $a_{1}=0.6, a_{2}=0.8, a_{3}=0.2$, $b_{1}=0.03, b_{2}=0.07, c_{1}=0.8, c_{2}=0.8, d_{1}=30, d_{2}=30, \mu_{1}$ $=0.4, \mu_{2}=0.4, \mu_{3}=0.08, \alpha=0.005, \beta=0.005, k_{1}=0.8$, $k_{2}=0.8, m=1, l=1$, and $p=10$. The bifurcation diagrams of $x_{1}(t), x_{2}(t)$, and $x_{3}(t)$ with respect to parameter $T$ in range $[107,155]$ are shown in Figure 4. Figures 4(a)-4(c) are bifurcation diagrams of $x_{1}(t), x_{2}(t)$, and $x_{3}(t)$, respectively.
From these diagrams, we can see that impulsive period $T$ heavily affects the dynamical behaviors. For example, Figure 5 shows the complex dynamic behaviors of $x_{3}$. Figure 5 is the magnified parts of Figure 4(c). From Figure 5(a), we can see the following: when impulsive period $107<T<117$, it is stable; if $T=117,142.4,148$, and 149.1, then bifurcation appears, respectively. That is to say, when $T>117$, there is a cascade of period-doubling bifurcations leading to $2 T$ period solution (Figure 5(a)); when $T>142.4$, 4T-period solution appears (Figure 5(a)); when $T>148$, 8T-period solution appears (Figure 5(b)); when $T>149.1,16 T$-period solution appears (Figure 5(b)). As $T$ increase beyond 149.5, the phenomenon of "crisis" emerges. When $T=151.6$, there is a typical chaotic oscillation (Figure 5(c)). When $T$ is near 153.5 , we can see in the neighborhood of $T=153.5$, after the period-doubling bifurcations, the symmetry-breaking bifurcations appear (Figure 5(d)), which are specially simple bifurcations that come into being multiplicity of steady states [5]. It implies that when $107<T<117$, that is, spraying 


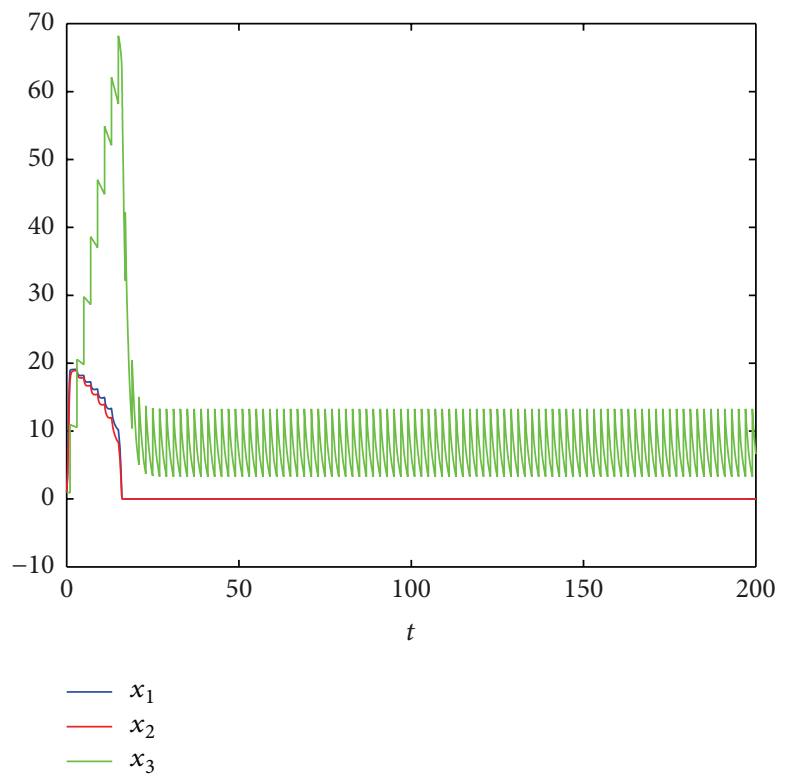

(a)

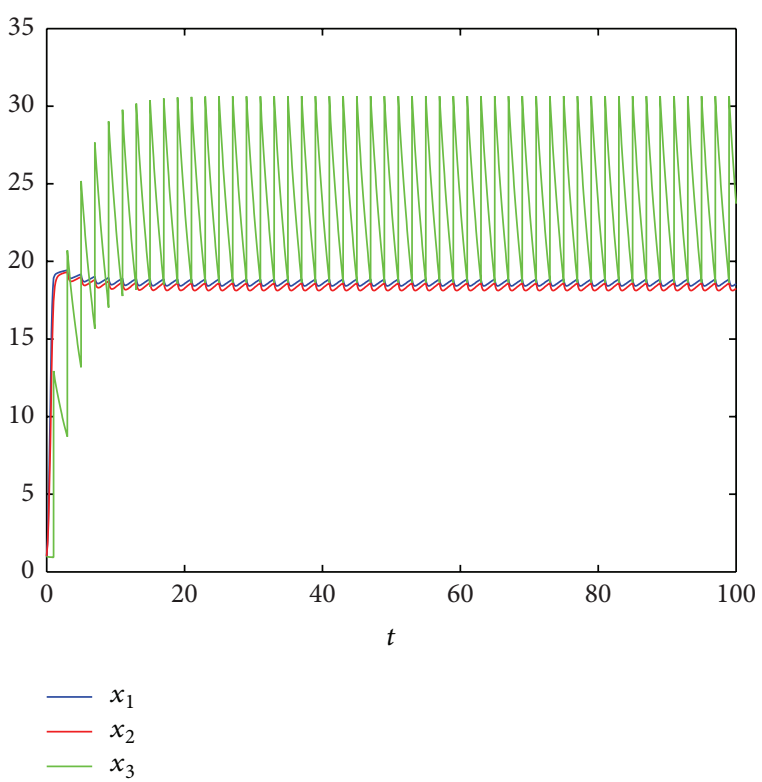

(b)

FiguRE 6: Dynamical behaviors of prey-eradication periodic solution and permanence of the system with single chemical control: (a) is the prey-eradication periodic solution of the single chemical control; (b) is the permanence of the single chemical control.

pesticides and releasing natural enemies are frequent, the solution of this system is stable in this situation; when $117<$ $T<149.5$, periodic behaviors of prey and the predator will appear; if $149.5<T<155$, then dynamical properties of this system are complex and the development of this system may be unpredictable.

Fourthly, let $x_{1}(t)=0.6, x_{2}(t)=1$, and $x_{3}(t)=1$ and $a_{1}=0.6, a_{2}=0.65, b_{1}=0.86, b_{2}=0.83, \mu_{1}=0.01, \mu_{2}=$ $0.01, \alpha=0.01$, and $\beta=0.01$. By verification, these parameters satisfy conditions of Corollary 11; then we can get the globally asymptotic stability of prey-eradication periodic solution and permanence for single chemical control (see Figures 6(a) and 6(b)).

\section{Conclusion}

In this paper, an impulsive two-prey and one-predator system with square root functional responses, mutual interference, and integrated pest management is constructed. Numerical simulations are given to portray the complex behaviors of this system. From Theorem 9, the existence and global asymptotic stability of prey-eradication periodic solution of (4) are obtained. Some methods and sufficient conditions are given to prove the permanence of system (4) in Theorem 10.

From Theorems 9 and 10 and simulations, we know dynamical properties of system (4) are very complex which depend on impulsive period $T$, the releasing amount of predator $p$, the mutual interference of predator $m$, and the parameter $\mu_{i}(i=1,2,3)$ of pests or predator which dies from the chemical control. Figures 1(c) and 3(c) show that different values of mutual interference of predator have different dynamical properties for system (4). Figures 1(a), 1(b), and 2 show the existence and attractivity of prey-eradication periodic solution. Figures 3(a) and 3(b) show the existence of positive periodic solution. Figure 4 implies that impulsive period $T$ heavily influences the dynamic behavior of system (4). As $T$ changes, periodic behaviors, bifurcations, "crisis" phenomenon, chaotic phenomenon, chaotic oscillation, and symmetry-breaking bifurcations appear, respectively. Hence, we can choose moderate value of $T$ for some different control strategies.

In this paper, by considering mutual interference and square root functional responses, our constructed model is new and complex, which more rationally reflects the real world. Furthermore, we also give the corresponding results on strategies of integrated pest management and classical chemical control. By our obtained results, if combining biological control and chemical control to eradicate preys, we can choose moderate impulsive period $T$ and moderate parameters $p$ and $\mu_{i}(i=1,2,3)$ to effectively eliminate preys and reduce environmental pollution. In particular, we analyze the influence from impulsive period $T$. These theories have some guidance to our real life and the natural balance.

\section{Competing Interests}

None of the authors has any competing interests in the paper.

\section{Acknowledgments}

This paper is supported by National Natural Science Foundation of China (11161015), Natural Science Foundation of Guangxi (2013GXNSFAA019003), Applied Statistics Key Laboratory (Guijiaokeyan (2014) number 6), and Statistics Guangxi Key Discipline (Guijiaokeyan (2013) number 13) and is partially supported by the National High Technology 
Research and Development Program 863 under Grant no. 2013AA12A402.

\section{References}

[1] Y. Shao, B. Dai, and Z. Luo, "The dynamics of an impulsive one-prey multi-predators system with delay and Holling-type II functional response," Applied Mathematics and Computation, vol. 217, no. 6, pp. 2414-2424, 2010.

[2] H. Baek, "Species extinction and permanence of an impulsively controlled two-prey one-predator system with seasonal effects," BioSystems, vol. 98, no. 1, pp. 7-18, 2009.

[3] W. M. Wang, H. L. Wang, and Z. Q. Li, "Chaotic behavior of a three-species Beddington-type system with impulsive perturbations," Chaos, Solitons \& Fractals, vol. 37, no. 2, pp. 438443, 2008.

[4] S. Zhang, D. Tan, and L. Chen, "Chaos in periodically forced Holling type II predator-prey system with impulsive perturbations," Chaos, Solitons and Fractals, vol. 28, no. 2, pp. 367-376, 2006.

[5] B. Liu, L. S. Chen, and Y. J. Zhang, "The dynamics of a preydependent consumption model concerning impulsive control strategy," Applied Mathematics and Computation, vol. 169, no. 1, pp. 305-320, 2005.

[6] Q. Wang, B. Dai, and Y. Chen, "Multiple periodic solutions of an impulsive predator-prey model with Holling-type IV functional response," Mathematical and Computer Modelling, vol. 49, no. 910, pp. 1829-1836, 2009.

[7] H. Wang and W. Wang, "The dynamical complexity of a Ivlev-type prey-predator system with impulsive effect," Chaos, Solitons Fractals, vol. 38, no. 4, pp. 1168-1176, 2008.

[8] V. Ajraldi, M. Pittavino, and E. Venturino, "Modeling herd behavior in population systems," Nonlinear Analysis: Real World Applications, vol. 12, no. 4, pp. 2319-2338, 2011.

[9] P. A. Braza, "Predator-prey dynamics with square root functional responses," Nonlinear Analysis: Real World Applications, vol. 13, no. 4, pp. 1837-1843, 2012.

[10] X. Ma, Y. Shao, Z. Wang, M. Luo, X. Fang, and Z. Ju, "An impulsive two-stage predator-prey model with stage-structure and square root functional responses," Mathematics and Computers in Simulation, vol. 119, pp. 91-107, 2016.

[11] M. P. Hassell, "Density-dependence in single-species populations," The Journal of Animal Ecology, vol. 44, no. 1, pp. 283-295, 1975.

[12] D. C. He, W. T. Huang, and Q. J. Xu, "The dynamic complexity of an impulsive Holling II predator-prey model with mutual interference," Applied Mathematical Modelling, vol. 34, no. 9, pp. 2654-2664, 2010.

[13] C. Zhang, N.-J. Huang, and D. O'Regan, "Almost periodic solutions for a Volterra model with mutual interference and Holling type III functional response," Applied Mathematics and Computation, vol. 225, pp. 503-511, 2013.

[14] Y. F. Shao and B. X. Dai, "The dynamics of an impulsive delay predator-prey model with stage structure and Beddington-type functional response," Nonlinear Analysis: Real World Applications, vol. 11, no. 5, pp. 3567-3576, 2010.

[15] Z. J. Liu and R. H. Tan, "Impulsive harvesting and stocking in a Monod-Haldane functional response predator-prey system," Chaos, Solitons \& Fractals, vol. 34, no. 2, pp. 454-464, 2007.

[16] P. Georgescu and H. Zhang, "An impulsively controlled pest management model with $\mathrm{n}$ predator species and a common prey," Bio Systems, vol. 110, no. 3, pp. 162-170, 2012.
[17] H. G. Yu, S. M. Zhong, R. P. Agarwal, and S. K. Sen, “Threespecies food web model with impulsive control strategy and chaos," Communications in Nonlinear Science and Numerical Simulation, vol. 16, no. 2, pp. 1002-1013, 2011.

[18] V. Lakshmikantham, D. D. Bainov, and P. S. Simeonov, Theory of Impulsive Differential Equations, World Scientific Publisher, Singapore, 1989.

[19] D. D. Bainov and P. S. Simeonov, Impulsive Differential Equations, vol. 28 of Series on Advances in Mathematics for Applied Sciences, World Scientific, Singapore, 1995.

[20] D. Bainov and P. Simeonov, Impulsive Differential Equations: Periodic Solutions and Applications, Longman Scientific and Technical, New York, NY, USA, 1993. 


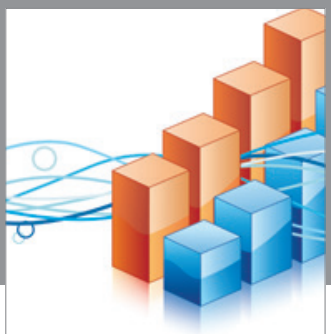

Advances in

Operations Research

vatem alat4

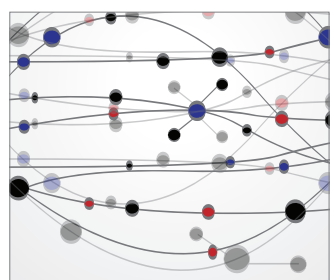

\section{The Scientific} World Journal
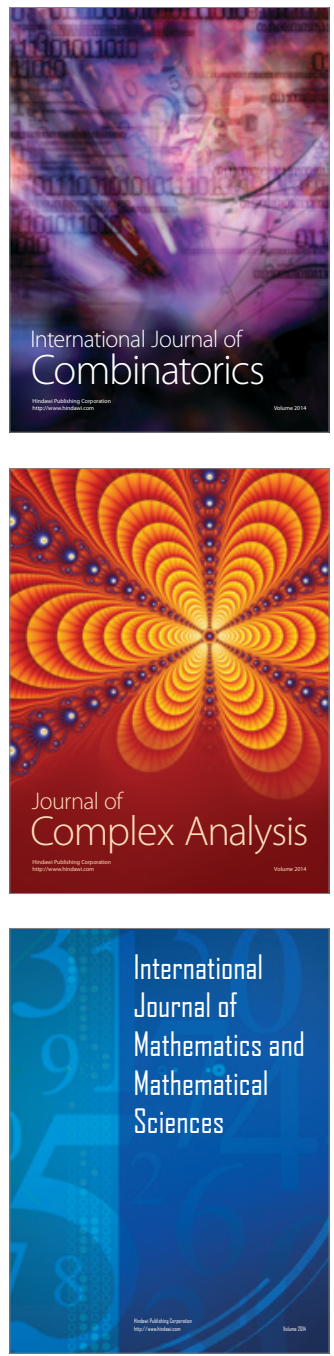
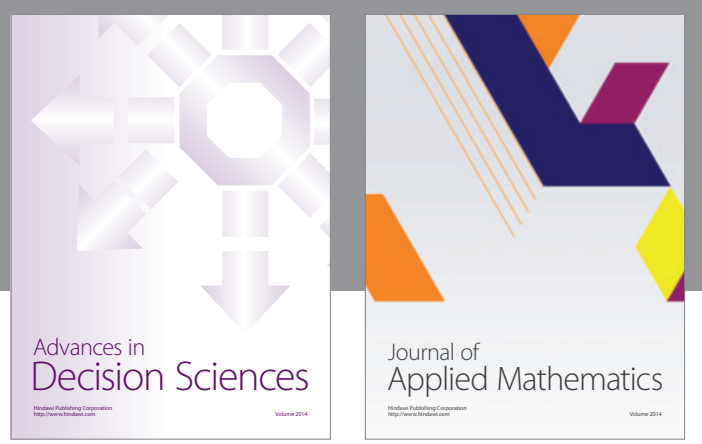

Algebra

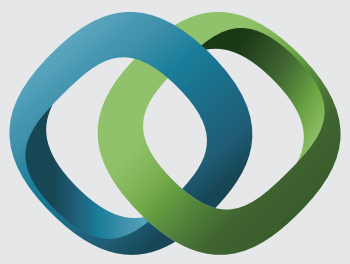

\section{Hindawi}

Submit your manuscripts at

http://www.hindawi.com
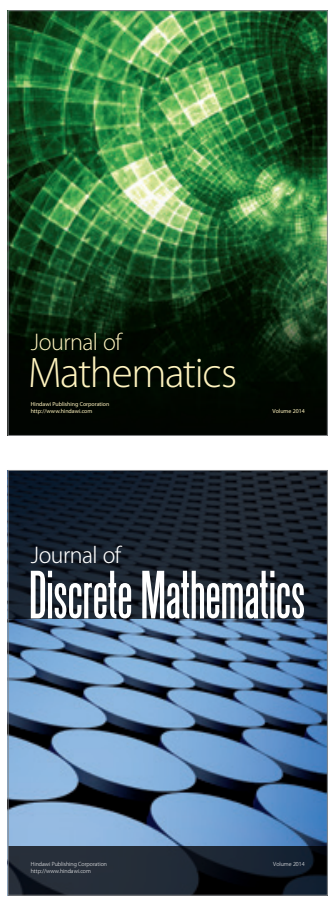

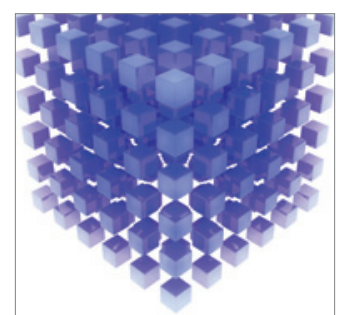

Mathematical Problems in Engineering
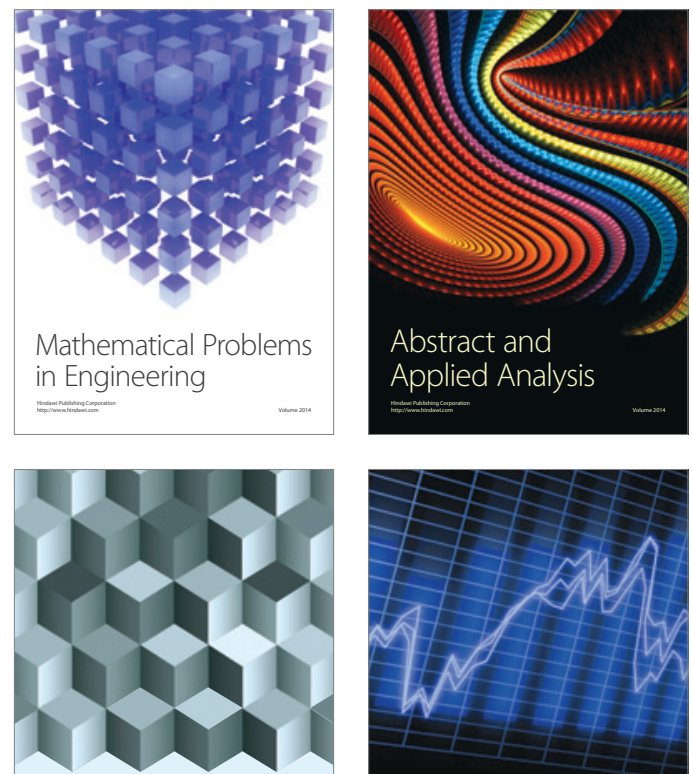

Journal of

Function Spaces

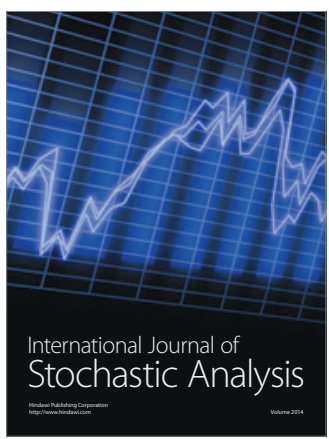

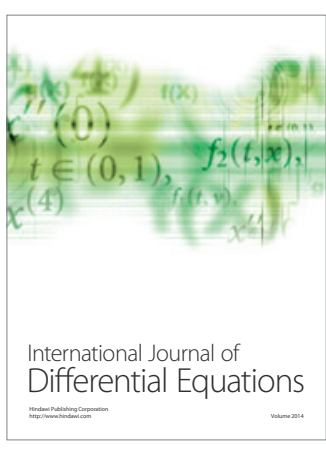
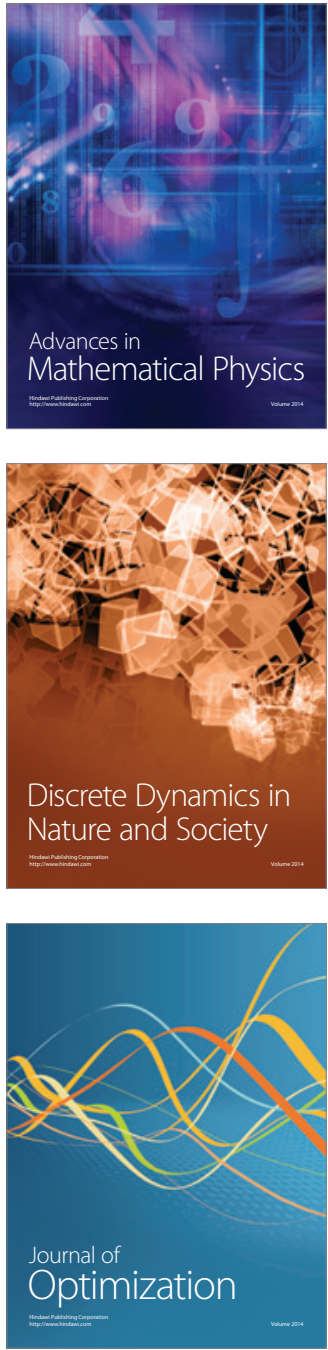